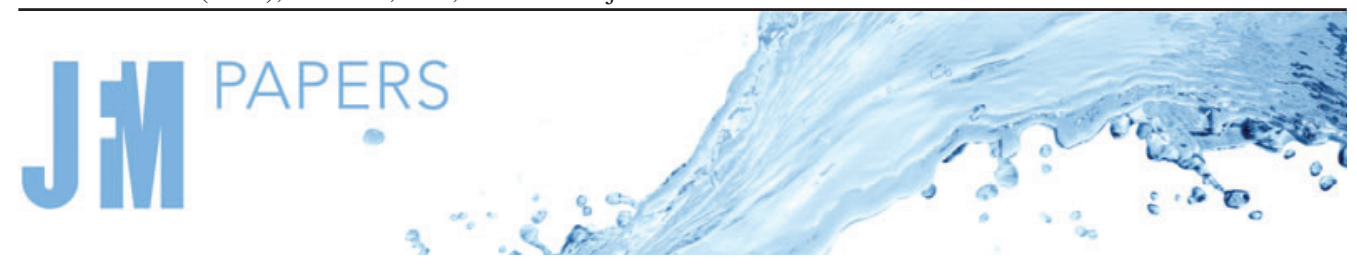

\title{
Shock induced aerobreakup of a droplet
}

\author{
Shubham Sharma ${ }^{1}$, Awanish Pratap Singh ${ }^{1}$, S. Srinivas Rao ${ }^{1}$, Aloke Kumar \\ and Saptarshi Basu ${ }^{1,2, \dagger}$ \\ ${ }^{1}$ Department of Mechanical Engineering, Indian Institute of Science, Bangalore 560012, India \\ ${ }^{2}$ Interdisciplinary Center for Energy Research (ICER), Indian Institute of Science, Bangalore 560012, \\ India
}

(Received 3 July 2021; revised 27 August 2021; accepted 27 September 2021)

The multiscale dynamics of a shock-droplet interaction is crucial in understanding the atomisation of droplets due to external airflow. The interaction phenomena are classified into wave dynamics (stage I) and droplet breakup dynamics (stage II). Stage I involves the formation of different wave structures after an incident shock impacts the droplet surface. These waves momentarily change the droplet's ambient conditions, while in later times they are mainly influenced by shock-induced airflow. Stage II involves induced airflow interaction with the droplet that leads to its deformation and breakup. Primarily, two modes of droplet breakup, i.e. shear-induced entrainment and Rayleigh-Taylor piercing (RTP) (based on the modes of surface instabilities) were observed for the studied range of Weber numbers (We 30-15000). A criterion for the transition between two breakup modes is obtained, which successfully explains the observation of RTP mode of droplet breakup at high Weber numbers $(W e \sim 800$ ). For $W e>1000$, the breakup dynamics is governed by the shear-induced surface waves. After formation, the Kelvin-Helmholtz waves travel on the droplet surface and merge to form a liquid sheet near the droplet equator. Henceforth, the liquid sheet undergoes breakup processes via nucleation of several holes. The breakup process is recurrent until the complete droplet disintegrates or external drag acting on the droplet is insufficient for further disintegration. At lower Weber numbers, the droplet undergoes complete deformation like a flattened disk, and a multibag mode of breakup based on RTP is observed.

Key words: drops and bubbles

\section{Introduction}

The atomisation of liquid droplets by aerodynamic forces is of great importance in many engineering and industrial applications. This process is widely exploited in the fields of

$\dagger$ Email address for correspondence: sbasu@iisc.ac.in

(C) The Author(s), 2021. Published by Cambridge University Press. This is an Open Access article, distributed under the terms of the Creative Commons Attribution licence (http://creativecommons.org/ licenses/by/4.0/), which permits unrestricted re-use, distribution, and reproduction in any medium, provided the original work is properly cited. 
liquid jet atomisation, agricultural spraying, combustion engines, energy systems, nuclear fusion processes and aerospace applications (Bayvel 1993; Eggers \& Villermaux 2008; Shinjo \& Umemura 2011; Lefebvre \& McDonell 2017). This topic has been extensively studied by researchers from incompressible to compressible flow regimes through the application of air-jet flow (Cao et al. 2007; Zhao et al. 2013) and shock tubes flow (Sun et al. 2005; Liang et al. 2020) for varying viscosities of Newtonian (Chang, Deng \& Theofanous 2013; Keshavarz et al. 2016; Yang \& Peng 2019) and non-Newtonian fluids (Joseph, Belanger \& Beavers 1999; Theofanous et al. 2012; Zhao et al. 2012). The varying morphologies in the droplet breakup process for shock tube studies are generally presented by means of deformation, breakup and secondary atomisation, depending on the droplet size and flow conditions (subsonic to supersonic). In this context, understanding the dynamic behaviour and breakup methodologies for different conditions is essential.

A wide range of experimental and numerical studies in the literature has investigated the droplet breakup mechanisms using the shock tube method. However, limited studies have emphasised the importance of early-stage shock wave interaction in the breakup analysis (Meng \& Colonius 2015; Sembian et al. 2016; Tanno et al. 2003; Sun et al. 2005). Sembian et al. (2016) used experimental and numerical techniques to explain the evolution dynamics of reflected, transmitted and diffracted waves during the interaction of an incident shock wave with a cylindrical water column. The possibility of cavitation in the droplet due to the expansion wave focusing at higher shock Mach numbers was observed. A numerical simulation by Meng \& Colonius (2015) and Guan et al. (2018) illustrated the development of recirculating flow near the equator region and an upstream jet at the droplet wake, which eventually assists in droplet deformation and breakup. Similar wave and flow dynamics were observed in the numerical simulation by Das \& Udaykumar (2020). In addition to deformable dispersions, several works were focused on understanding the wave dynamics of a planar shock wave interaction with solid dispersions (Tanno et al. 2003; Sridharan et al. 2015; Mehta et al. 2016, 2018). Tanno et al. (2003) studied the interaction with a solid sphere and used holographic double exposure interferometry to show that the sphere experiences the maximum drag force during the transition of reflection wave from regular to Mach reflection. The work of Sridharan et al. (2015), Mehta et al. (2016) and Mehta et al. (2018) was focused on understanding the variation of drag coefficient experienced by a particle with varying interparticle distances in a random (Mehta et al. 2018), inline (Sridharan et al. 2015) and transverse orientation (Mehta et al. 2016).

Subsequently, the dependence of droplet breakup mode with Ohnesorge number $(\mathrm{Oh}=$ $\left.\mu_{l} / \sqrt{\rho_{l} \sigma D_{i}}\right)$ and Weber number $\left(W e=\rho_{a} V_{i}^{2} D_{i} / \sigma\right)$ was specified by Hinze (1955) which was further extended by Krzeczkowski (1980) to mark the transition points on the $W e-O h$ regime. Here, $\mu_{l}$ and $\rho_{l}$ are dynamic viscosity and density of droplet, $\sigma$ is the surface tension of the air-liquid interface, $D_{i}$ is the initial droplet diameter before shock interaction, $\rho_{a}$ and $V_{i}$ are the density and velocity of airflow post shock conditions. The Weber number accounts for relative dominance of aerodynamic/inertial force to the surface tension force, while the Ohnesorge number accommodates the effect of liquid viscosity on breakup dynamics. For viscoelastic fluids and viscous Newtonian fluids, the deformation and breakup of a droplet through bag and stamen process at higher Weber numbers is reported in the study of Joseph et al. (1999). The increasing Oh values lead to retardation in the deformation and breakup process, while negligible influence of viscosity was observed for $O h<0.1$ (Hsiang \& Faeth 1992). Numerous reviews consolidated the droplet fragmentation modes and corresponding breakup transition criteria (Pilch \& Erdman 1987; Guildenbecher, López-Rivera \& Sojka 2009; Theofanous 2011). Primarily, five modes of aerodynamic breakup of a droplet, i.e. bag breakup $(24>W e>11)$, 
bag-stamen/plume breakup $(65>$ We $>24)$, multibag breakup $(85>W e>65)$, sheet thinning/stripping breakup $(120>$ We $>85)$ (Jain et al. 2015) and catastrophic breakup $(W e>350)$ (Guildenbecher et al. 2009) are obtained for $\mathrm{Oh}<0.1$ based on different flow Weber number values. The breakup phenomenon in the direction of air-jet flows with the formation of multimode structures due to Rayleigh-Taylor instability (RTI) at lower We of 15-40 was studied by Cao et al. (2007) and Zhao et al. (2013). The transition of various breakup modes to the shear breakup regime with the formation of liquid sheets at the equator along with the effect of viscosity and density ratios were presented by Chou, Hsiang \& Faeth (1997), Han \& Tryggvason (2001) and Jain et al. (2015). Similarly, shear stripping of the secondary droplets with the impact of a shock wave was visualised by Nicholls \& Ranger (1969), Chou et al. (1997), Theofanous \& Li (2008), Jalaal \& Mehravaran (2014), Meng \& Colonius (2015), Biasiori-Poulanges \& El-Rabii (2019) and more recently by Wang et al. (2020) and Dorschner et al. (2020). Nicholls \& Ranger (1969) showed that the droplets' breakup mainly occurs due to the induced airflow behind the shock wave, while Chou et al. (1997) focused on identifying size and velocity distribution of fragmented droplets.

Theofanous \& Li (2008) briefed that the previously identified catastrophic breakup regime was an artefact of experimental techniques used in such works. Biasiori-Poulanges \& El-Rabii (2019) used high-magnification and high-speed breakup images of interaction to demonstrate the evolution dynamics of the breakup process at a higher Weber number of 540-1350. Further, subsonic to supersonic velocity of induced flow was considered by Wang et al. (2020) for identifying the individual effects of shock Mach number $\left(M_{S}\right)$ and Reynolds number $(R e)$ on breakup dynamics while holding the Weber number as a constant. Recently, Dorschner et al. (2020) carried out a three-dimensional simulation on a droplet exposed to high-speed airflow to show the ligament formation and shedding as functions of Weber number and vortex shedding at the droplet wake. The above discussed different breakup modes were further reclassified (Theofanous \& Li 2008; Theofanous 2011; Theofanous et al. 2012) based on hydrodynamics instabilities responsible for such breakups. In this context, for We $<100$, the previously identified bag, bag-stamen and multibag breakup modes, were consolidated as the Rayleigh-Taylor piercing (RTP) mode (based on RTI). Similarly, at higher Weber numbers $(W e>1000)$ previously identified sheet thinning/stripping and catastrophic modes were consolidated as a shear-induced entrainment (SIE) mode (based on Kelvin-Helmholtz instability (KHI)). The same description for the two modes is used in the present work.

The hydrodynamic instabilities, primarily the RTI, KHI and Rayleigh-Plateau instability (RPI) play a crucial role in predicting the droplet morphology during aerodynamic atomisation of a droplet. Historically RTI is shown to be the driving cause for the breakup modes involving bag formation (Zhao et al. 2010; Theofanous 2011; Jain et al. 2015), where different bag-type breakups are predicted based on the dimensionless parameter $\left(N=D_{i} / \lambda_{R T}\right)$. Here, $\lambda_{R T}$ is the Rayleigh-Taylor (RT)-based instability wavelength. The existing literature shows the existence of bag-breakup for $N=1 / \sqrt{3}-1$, bag-stamen for $N=1-2$ and multibag mode of breakup for $N>2$ (Zhao et al. 2010; Jain et al. 2015), where the RTI wavelength is predicted from conventional RT analysis (Rayleigh 1879). Recently, Theofanous (2011) and Theofanous et al. (2012) used finite thickness RTI analysis for a two-phase system to identify the condition for the first criticality, i.e. bag formation process. In contrast to the bag-type mode of droplet breakup, the shear-induced breakup of a droplet is driven by the KHI on the droplet surface (Theofanous \& Li 2008; Theofanous et al. 2012; Jalaal \& Mehravaran 2014; Liu et al. 2018; Biasiori-Poulanges \& El-Rabii 2019; Wang et al. 2020). 
Here Kelvin-Helmholtz (KH) waves are augmented by the action of shear on the liquid-air interface. Although this mechanism of droplet breakup at high Weber number (We > 1000) is discussed in many works, an actual attempt to compare the formed KHI waves with the theoretical analysis is still limited (Marmottant \& Villermaux 2004; Kim et al. 2006; Theofanous et al. 2012; Jalaal \& Mehravaran 2014) and most of which are primarily numerical (Kim et al. 2006; Jalaal \& Mehravaran 2014) or seminumerical (Theofanous et al. 2012), while the experimental works are related to liquid-air interaction in a coaxial jet system (Marmottant \& Villermaux 2004). Further, the RPI mode of instability was observed in the secondary atomisation of formed ligaments during the breakup of the primary droplet (Biasiori-Poulanges \& El-Rabii 2019). All these modes of interfacial instabilities are explained in the context of aerodynamic atomisation in the present work.

The aerodynamic breakup of a liquid droplet is a high-speed phenomenon where the droplet disintegration is completed within the time scales of several hundred microseconds, particularly at higher Weber numbers $(W e>1000)$. A high-speed interaction dynamics is difficult to perceive through numerical and experimental means due to short time scales and the multiscale nature of the breakup phenomenon. The disintegration of a millimetre-sized primary droplet into micron-sized daughter droplets within a time scale of few microseconds requires high spatiotemporal resolution. Although numerical advancement in this direction can be achieved by increasing the computational cost (Poplavski et al. 2020), and has also been attempted much efficiently in recent times (Jalaal \& Mehravaran 2014; Meng \& Colonius 2015; Guan et al. 2018; Liu et al. 2018; Dorschner et al. 2020), the experimental works pertaining to high spatiotemporal resolution are still lacking. For example, a high-speed camera's field of view must be compromised to increase the temporal resolution. In addition to this, the spatial resolution has to be further compromised to accommodate the possible uncertainties in the falling droplet and incoming shock wave locations between the experimental runs. The usage of high exposure times (even within microsecond order) results in the motion blur of fast-moving atomised droplets, which can lead to a misinterpretation of the actual phenomenon (as discussed by Theofanous \& Li (2008)). Correspondingly, the high-speed breakup dynamics of the liquid droplet is essentially a three-dimensional phenomenon, thereby necessitating the interaction observations through multiple viewing angles, further increasing the experimental complexities. Therefore, a highly sophisticated and optimised experimental arrangement is required for effectively capturing the multiscale nature of the droplet's aerodynamic breakup and involved physical phenomenon. Several experimental studies in the recent literature have attempted some of these aspects (Theofanous \& Li 2008; Biasiori-Poulanges \& El-Rabii 2019; Jackiw \& Ashgriz 2021); however, a more comprehensive study providing benchmark measurement data for numerical simulation, involving a wide parametric range and covering complete evolution dynamics of interaction phenomenon is still lacking.

From a physics perspective, earlier works were focused on identifying the various modes of droplet breakup, primarily at low Weber numbers (We <250) (Guildenbecher et al. 2009; Zhao et al. 2010; Jain et al. 2015; Zhao et al. 2018) and predicting various breakup modes using stability analysis. At higher Weber numbers (We $>1000)$ observations are primarily made through numerical simulations (Jalaal \& Mehravaran 2014; Guan et al. 2018; Liu et al. 2018; Biasiori-Poulanges \& El-Rabii 2019; Dorschner et al. 2020). However, most of these works lack the accurate prediction of the atomisation stage, requiring high spatiotemporal resolutions. Several experimental studies have been reported in this regime (Theofanous \& Li 2008; Theofanous et al. 2012; Biasiori-Poulanges \& El-Rabii 2019; Dorschner et al. 2020; Wang et al. 2020) which primarily focused on identifying the evolution of the droplet breakup process in the SIE regime. However, a 


\section{Shock induced aerobreakup of a droplet}

clear understanding of the three-dimensional breakup process is still lacking, and limited attempts have been made (Theofanous et al. 2012; Biasiori-Poulanges \& El-Rabii 2019) for comparing the observed surface instabilities with theoretical analysis. The existing literature shows a monotonous dependence of breakup mechanism on Weber number, i.e. breakup mode changes from RTP to SIE with the increase in Weber number. However, in the present work, by using submillimetre to millimetre-sized deionised water droplets, we obtained an essential criterion that shows that RTP can exist at a high Weber number of $\sim 800$. Similarly, SIE can exist at lower $W e \sim 200$, thereby exhibiting a non-monotonous dependence on Weber number.

In this context, the present study addresses the full interaction dynamics of a liquid droplet interacting with a shock wave of varying Mach numbers ( $M_{S} \sim 1.1$ to 1.8 ). The effect of aerodynamic breakup on different droplet sizes $\left(D_{i}=0.5,2.5\right.$ and $\left.2.9 \mathrm{~mm}\right)$ is studied by employing visualisation techniques such as schlieren and shadowgraphy to understand the significant flow features and structural morphologies during the regimes of deformation and disintegration of the droplet. The experimental set-up of the shock tube is further simplified in design for generation of the required shock wave through the exploding wire technique. The sophisticated and optimised experimental arrangement used in the present work addressed the concern of high spatial-temporal imaging of interaction phenomena. The initial shock interaction flow features were quantified in detail for the local flow conditions and the possible deformation shapes depending on the size of droplet and shock Mach numbers. A wide range of Weber number values (We 30-12 000) resulted in the observation of both RTP and SIE modes of droplet breakup. An essential criterion for the transition of breakup modes is also discussed.

This paper is organised as follows. Section 2 provides the details of experimental set-up and methodology used, the results and discussion of this work, which involves explanations on the shock-droplet interaction mechanism ( $\$ 3.1)$, global observation of phenomena ( $\$ 3.2)$, SIE breakup ( $\$ 3.3)$, KH waves surface instability ( $\$ 3.4)$ and breakup mode transition mechanism $(\S 3.6)$, are shown in $\S 3$. The conclusions of the present study are provided in $\S 4$.

\section{Experimental set-up}

The interaction of a freely falling droplet with a shock wave is carried out in a specially designed shock tube apparatus based on the exploding wire technique, which offered the possibility to operate at the desirable shock Mach numbers $\left(M_{S}\right)$ ranging from 1.1 to 1.8 . A detailed overview of the exploding wire technique and its application in shock wave generation can be found elsewhere (Fedotov-Gefen et al. 2010; Liverts et al. 2015; Sembian et al. 2016). This technique offers several advantages compared with diaphragm-based shock tubes, such as small size test facilities, ease of operation, generation of a wide range of shock strengths $\left(M_{s}=1-6\right)$ (Sembian et al. 2016) and high repeatability between tests. A schematic representation of the experimental test cavity is shown in the figure $1(a)$. Two separate flow channels (rectangular) are designed with dimensions of $360 \mathrm{~mm} \times$ $50 \mathrm{~mm} \times 20 \mathrm{~mm}$ and $400 \mathrm{~mm} \times 50 \mathrm{~mm} \times 10 \mathrm{~mm}$, resulting in a wide range of shock Mach numbers. The sidewalls of the flow channel and the cover plates of the exploding chamber are fabricated using polycarbonate due to its high impact strength, while acrylic cover plates are used on the remaining length (figure $1 a$ ). The cover plates are firmly attached with screws to prevent compressed air from escaping. The shock tube is attached to the electrode chamber (by a nut and bolt arrangement), where the copper wire (of $35 \mathrm{SWG}$ ) is securely placed between two electrodes. A high-voltage power supply is connected to the two electrodes to accomplish the wire-blast system. 
(a)

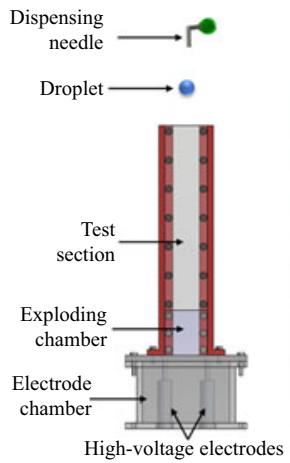

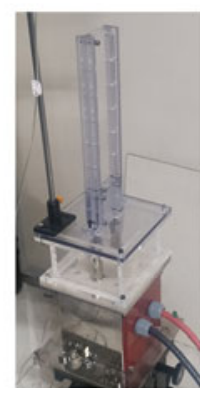

(b)

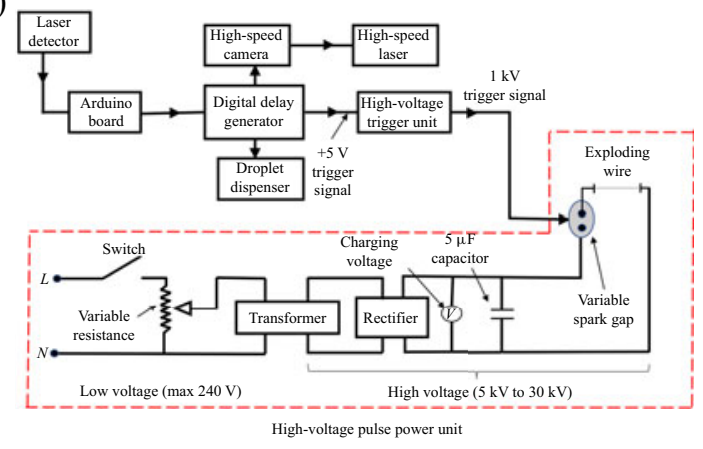

Figure 1. Exploding-wire based shock tube set-up. (a) Schematic diagram of shock tube and actual shock tube tower; and $(b)$ electrical wiring diagram for high-voltage pulse power unit and triggering set-up.

The wiring diagram (simplified version) for the management of the $2 \mathrm{~kJ}$ high-voltage pulse power system (Zeonics Systech, India (Z/46/12)), which is used for the wire explosion and synchronisation of the various instruments for the experimentation process, is shown schematically in figure $1(b)$. For the generation of a high-voltage pulse, the incoming $\mathrm{AC}$ power supply is altered to the required voltage range of $5 \mathrm{kV}$ to $12 \mathrm{kV}$ through a step-up transformer. The high voltage AC power supply is then converted to DC power with a rectifier arrangement which is then further used for charging a $5 \mu \mathrm{F}$ capacitor. Once the charging of the capacitor to the desired energy level is complete, the charging circuit is cut off, and the discharging circuit connected to the exploding wire is closed by providing a $1 \mathrm{kV}$ trigger signal from the high-voltage trigger unit (which is activated by a trigger signal from the digital delay generator) at the variable spark-gap switch. Once the unit is triggered, a high-voltage pulse is discharged from the electrodes and passes through a thin copper wire, which results in rapid Joule's heating, vaporisation of the wire and generation of a cylindrical blast wave, which is called the exploding-wire technique. The induced blast wave inside the rectangular column is transformed to a planar shock by the narrow geometry of flow channel (Sembian et al. 2016). The formed shock wave interacts with the water droplet at a distance of $15 \mathrm{~mm}$ from the column exit point in open ambient conditions. This configuration is chosen due to several limitations of doing experiments inside the shock tube channel, such as the sticking of stripping droplets on cover plates during droplet breakup, which hinders the visualisation of the interaction phenomena and formation of reflected waves from the inner walls of the shock tube. Further, no significant difference is observed in the shock wave dynamics carried out inside and outside of the shock tube (see supplementary movie 1 available at https://doi.org/10.1017/jfm.2021.860). The synchronisation of droplet ejection, its interaction with a shock wave and simultaneous capturing of interaction phenomena are done within nanosecond accuracy using a BNC $745 \mathrm{~T}$ digital delay generator.

The deionised water droplet dispensing system is aligned above and at a central position of the shock tube cavity. The ejected droplet diameter ranges from $2.9 \mathrm{~mm}$ to $0.5 \mathrm{~mm}$, in which a syringe pump (New Era Pump Systems, NE-1010) based droplet ejection is used for droplet sizes greater than $1 \mathrm{~mm}$, and a Nordson PICO P $\mu l$ se dispensing system is used for sizes less than $1 \mathrm{~mm}$. The syringe pump ejects a precise volume of droplet/fluid during the infusion of the syringe with precise rotation of the stepper motor, while the Nordson 
(a)

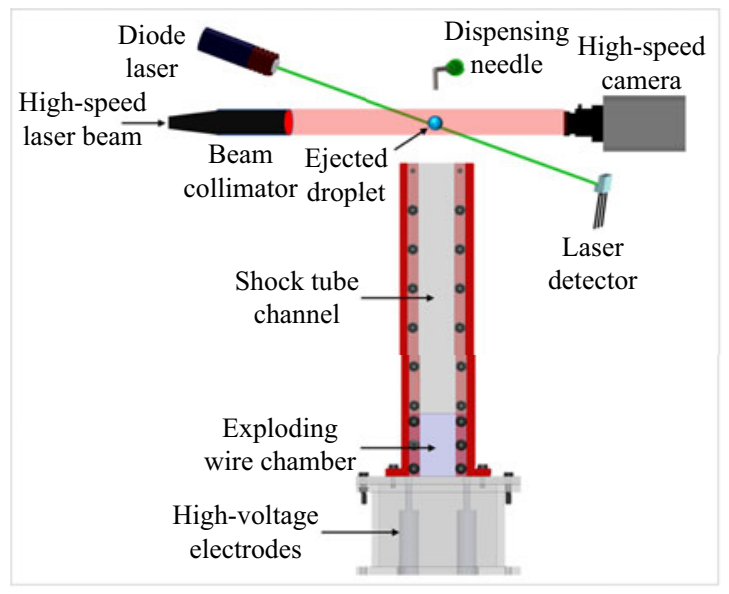

(b)

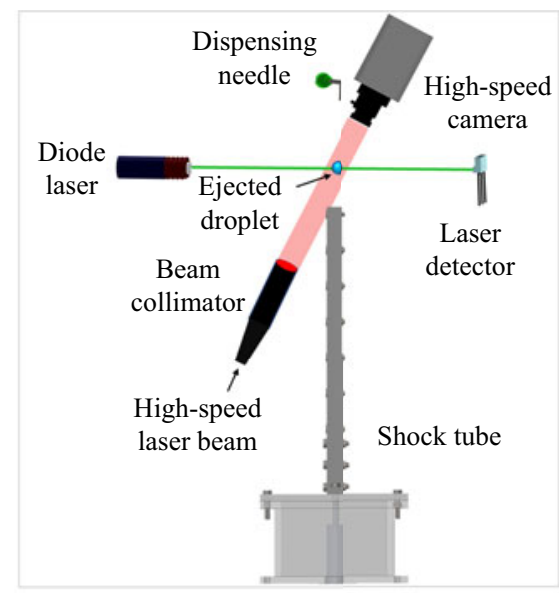

(c)

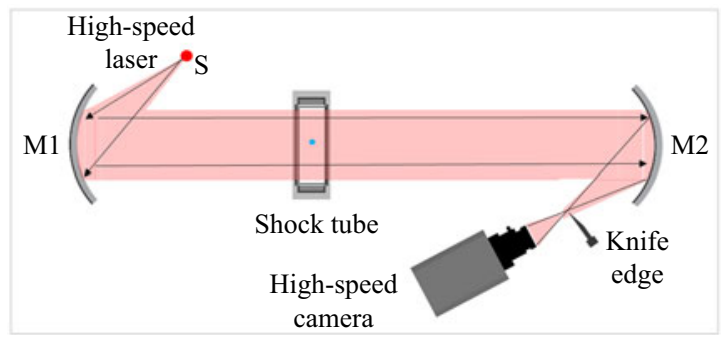

Figure 2. Experimental arrangement and imaging set-up. (a) Shadowgraphy imaging and laser interrupter set-up; $(b)$ inclined top-view imaging set-up; and $(c)$ schlieren imaging set-up.

PICO Pulse dispenser uses a piezoelectric-based precise droplet ejection mechanism. The free-falling droplet is detected at a specific position from the ejection point by its obstruction to a laser detector system, which is placed in the pathline of the falling droplet as shown in figure 2(a). Detected obstruction of the laser beam is used as a trigger signal to the digital delay generator as presented in the schematic view in figure 1(b). A high-speed pulse laser system, high-speed camera, high-voltage unit and a droplet dispenser unit are connected to the digital delay generator to trigger all the components at once with initial delay time intervals, which is operated from the external source (i.e. a personal computer). As the experiments are to be operated at microsecond time scales, the integration of all the components is vital for the experimentation to attain the subsequent droplet breaking phenomenon from initial drop ejection to shock wave generation, interaction, deformation and secondary atomisation of the droplet. The phenomenon of the droplet breakup process are essentially recorded through three imaging techniques such as shadowgraph imaging, top-view imaging and schlieren imaging.

Interaction of the shock wave and droplet breakup process for varying droplet sizes are captured by employing the shadowgraph technique as shown in figure 2( $a$ ). A high-speed and non-coherent pulse diode laser of $640 \mathrm{~nm}$ wavelength (Cavitar Cavilux smart UHS, $400 \mathrm{~W}$ power) is used for capturing the droplet breakup process at the laser pulse width of 10-40 ns. The use of nanosecond-scale pulse duration of a light source is essential for freezing the high-speed interaction phenomena. The emitted light beam is transformed into 
a parallel light beam using a collimator lens (Thorlabs, BE20M-A). A high-speed camera (Photron, SA5) is aligned in line to the beam source as shown in figure 2(a) which captures the shadow image of the disintegrating droplet during its interaction with the shock wave. The high-speed pulse laser source is synchronised with the high-speed camera for all frame rates ranging from 20000 to 100000 frames per second (f.p.s). The experimental images have been captured using the shadowgraph technique with extensive zoom-in and zoom-out views of the interaction to study the droplet surface instabilities and whole-field droplet breakup processes at different shock Mach numbers and droplet sizes. Further, to capture the breakup process in top view, the high-speed laser and camera system is aligned at a 20-degree orientation with the vertical axis using the assistance of heavy-duty tripod stands as shown in figure $2(b)$. The top-view arrangement is essential for an augmented understanding of the three-dimensional breakup process, as will be discussed later in $\S 3.5$. The characteristics of the droplet and its breakup process can be assessed explicitly using shadowgraphy, whereas the initial shock wave interaction phenomenon and flow field dynamics around the droplet are quantified by using the schlieren technique.

The alignment of the schlieren system is presented schematically in figure 2(c). The emitted beam from the pulse laser source is transformed to a point light source using variable round aperture (Holmarc SSID-25). This aperture acts as a point light source for the concave mirror (M1) oriented at its focal length, forming a parallel light beam. The test cavity is aligned in the path of a parallel beam for recording the shock-droplet interaction phenomena. Further, the light beam passes through the second spherical concave mirror (M2) and is focused to a point at the focal length of the mirror where a knife edge is positioned to block half of the incoming light in order to attain the variation of the density gradients during the interaction phenomena. This arrangement facilitates visualising complicated wave structures and ambient fluid flow features around the droplet during the initial interaction stages.

In the present work, the experiments are performed on different shock-Mach numbers $\left(M_{s}=V_{s} / c\right)$ ranging from 1.1 to 1.85 by the application of 5 to $12 \mathrm{kV}$ of charging voltage $\left(V_{c}\right)$ to the capacitor and two separate shock tube channels (see figure $3 a$ ). Here, $V_{s}$ is the shock wave velocity, and $c$ is the speed of sound in the medium ahead of the shock wave. The shock wave velocity is estimated by measuring the distance travelled by the shock wave in the two consecutive images recorded at $0.1 \mathrm{M}$ f.p.s. A comparison of this instantaneous shock velocity with the average velocity of the shock wave measured between two distant locations in the camera field is also made, which shows a negligible difference between the two results, indicating a minimal loss of shock strength during the studied time duration. Primarily, the shock velocity is a direct function of the applied charging voltage, as can be seen from figure 3(a). On the basis of obtained shock Mach numbers and the induced flow velocities (Anderson 2003), Weber number and Reynolds number $\left(\operatorname{Re}=\rho_{a} V_{i} D_{i} / \mu_{a}\right)$ for varying droplet diameters $(2.9 \mathrm{~mm}$ to $0.5 \mathrm{~mm}$ ) are estimated (see figure $3 b, c)$. Here, $\mu_{a}$ is the dynamic viscosity of the induced flow post shock conditions. The Weber number and Reynolds number in the present work ranges from $\sim 30$ to 12000 and $\sim 3000$ to 100000 , respectively. Droplets are dispensed in such a manner that their spherical shape is maintained. However, a slight deviation from the sphericity is observed, which was found to be $0.945 \pm 0.04,0.972 \pm 0.02$ and $0.911 \pm 0.06$ for droplet size of $2.9 \mathrm{~mm}, 2.5 \mathrm{~mm}$ and $0.5 \mathrm{~mm}$, respectively. A completely spherical droplet will have its roundness value of unity. The results of the observed interaction dynamics are presented in the following sections. 

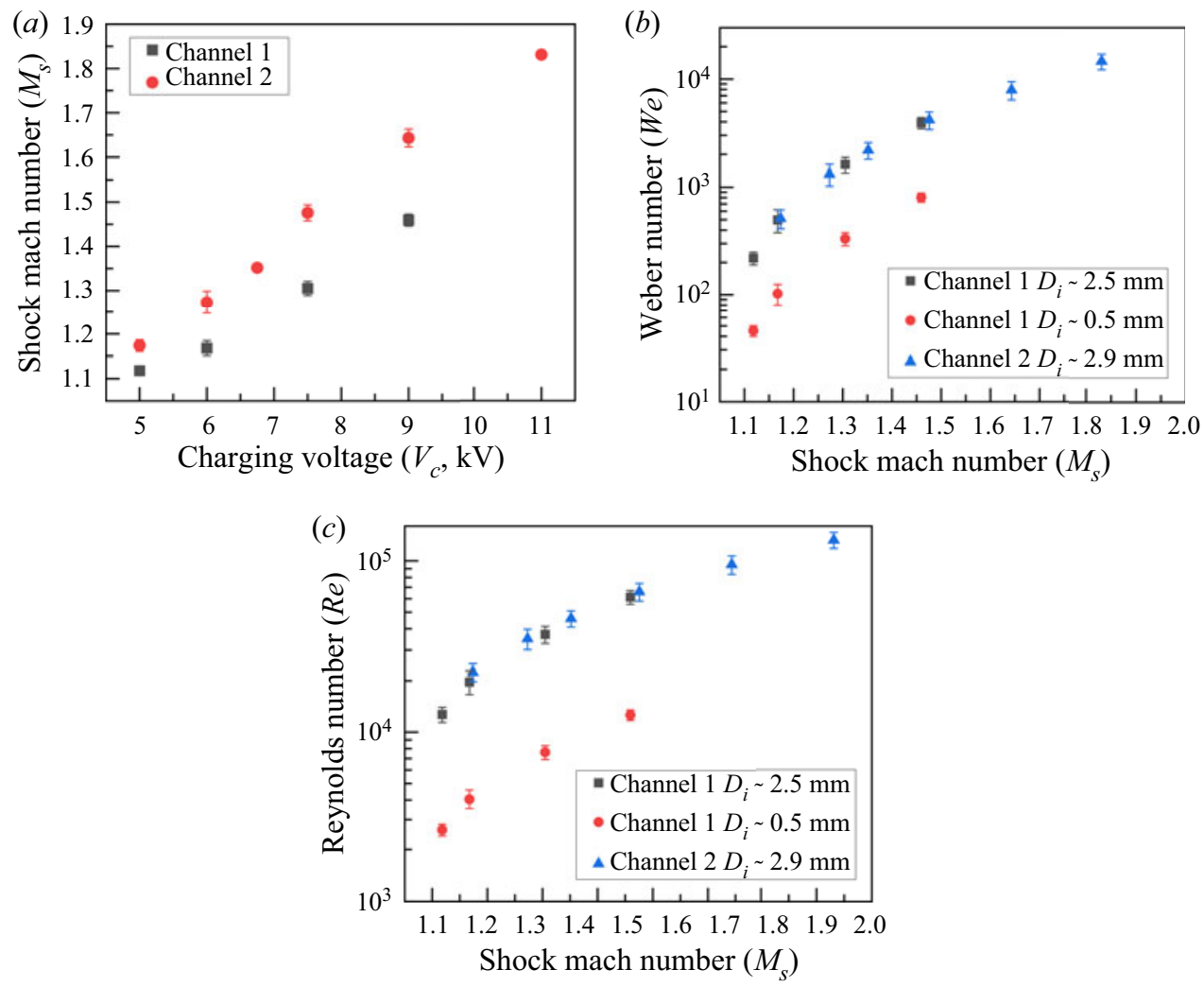

Figure 3. Range of non-dimensional parameters for channel 1 and channel 2. (a) Shock Mach number $\left(M_{S}\right)$ versus charging voltage $\left(V_{c}\right)$ in kilo-Volts; $(b)$ Weber number $(W e)$ versus shock Mach number $\left(M_{s}\right)$ for different droplet sizes $\left(D_{i} \sim 2.9,2.5 \mathrm{~mm}\right.$ and $\left.\sim 0.5 \mathrm{~mm}\right)$; and $(c)$ Reynolds number $(R e)$ versus shock Mach number $\left(M_{s}\right)$ for different droplet sizes $\left(D_{i} \sim 2.9,2.5 \mathrm{~mm}\right.$ and $\left.\sim 0.5 \mathrm{~mm}\right)$.

\section{Results and discussions}

\subsection{Shock-droplet interaction mechanism}

The interaction of an incident shock wave with a droplet surface leads to different wave structures like reflected wave, transmitted wave, Mach-stem and diffracted wave, which affects the flow conditions around the droplet periphery. Although these features are physically present, some features like transmitted wave, external and internal flow field of a droplet are difficult to perceive using currently available experimental techniques. The transmitted wave cannot be visualised due to the limitation of shadowgraphy and/or schlieren imaging techniques and due to the spherical shape of the droplet which acts as a lens during shadowgraphy/schlieren imaging. The flow dynamics could not be obtained experimentally due to the requirement of a high-resolution and high-frame-rate particle image velocimetry system (minimum requirement of 20000-40 000 f.p.s.), which necessitates the usage of a high-power pulse-burst laser system (Beresh et al. 2015). In addition, there are several challenges associated with seeding particles in supersonic and shock tunnel flows (Havermann et al. 2008). However, due to advancements in numerical methods and existing computational power, it is now possible to observe these features using numerical techniques as reported recently by several researchers (Chen 2008; Sembian et al. 2016; Sridharan et al. 2016; Guan et al. 2018; Das \& Udaykumar 
(a)

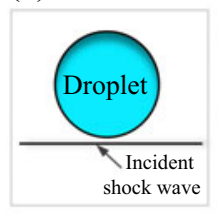

(b)

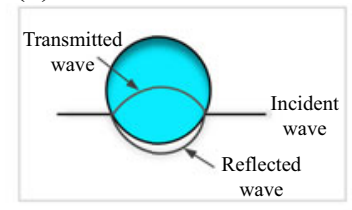

(e)

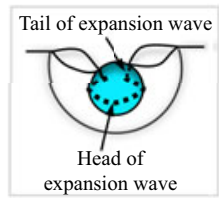

(i)

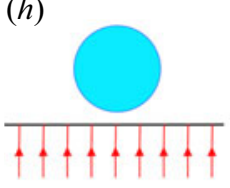

$(l)$

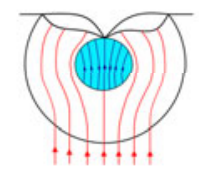

(c)

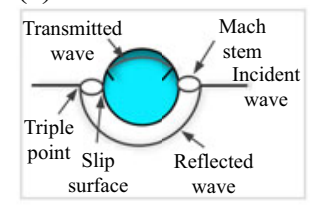

$(g)$ (d)

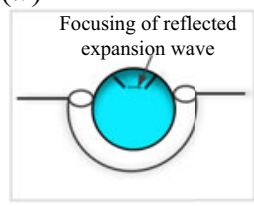

Figure 4. Shock-droplet interaction mechanism. $(a-g)$ Shock wave dynamics during different time instances; and $(h-o)$ external (red lines) and internal (blue lines) flow streamlines for a droplet at different time instances.

2020; Poplavski et al. 2020). Therefore, the discussion in the current subsection is based on the experimental observations of the present work and some flow features established in the above numerical studies. Figure 4 schematically shows the shock dynamics (figure $4 a-g$ ) and flow dynamics (figure $4 h-o$ ) realised during shock-droplet interaction. Figure 5 presents the schlieren images recorded at $0.42 \mathrm{M}$ f.p.s. with a pixel resolution of $64 \times 96$ pixels and corresponds to the features shown in figure 4 .

The incident shock interaction on the droplet surface creates a reflected wave, a transmitted wave and a diffracted wave. Depending on the acoustic impedance $(Z=\rho c)$ of the medium on either side of the interface, the reflected wave can be a shock wave or an expansion wave (Henderson 1989). It is interesting to note that if the medium through which the wave has travelled before reflection has a lower impedance than the medium on the other side of the reflecting surface, the reflected wave will be a shock wave and vice versa. For the current case, the reflected wave will be a shock wave $\left(Z_{\text {water }}>Z_{\text {air }}\right)$ (figures $4 b, i$ and $5 b$ ). The reference time $(t)$ is measured from the first instance of the shock interacting with the droplet surface. The reference time is non-dimensionalised using the inertial time scale $\left(\tau=D_{i} / V_{i} \sqrt{\rho_{l} / \rho_{a}}\right)$ as $t^{*}=t / \tau$. The planar incident wave will undergo regular reflection until the incidence angle $\left(\alpha_{i}\right)$ is greater than the critical detachment angle $\left(\alpha_{c}\right)$ (Ben-Dor 2007). If $\alpha_{i}<\alpha_{c}$, a regular reflection is not possible and transition to Mach reflection occurs (figures $4 c, j$ and $5 c$ ). A triple point is formed at the intersection of the incident wave, reflected wave and Mach stem. A slip surface is also formed at the intersection of the Mach wave and droplet surface. A uniform stream of induced airflow 
(a) $t^{*}=0$

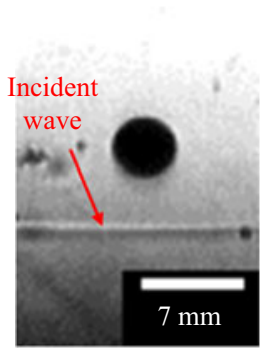

(b) $t^{*}=0.01$

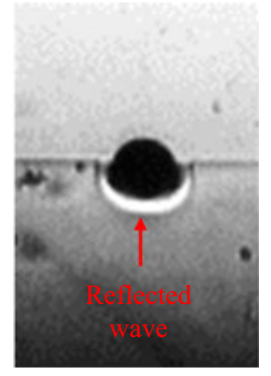

(c)

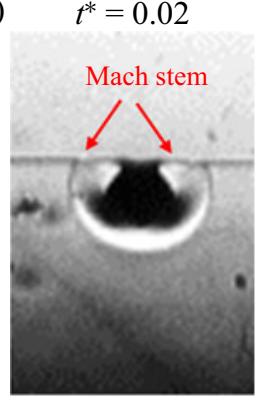

(d) $t^{*}=0.04$

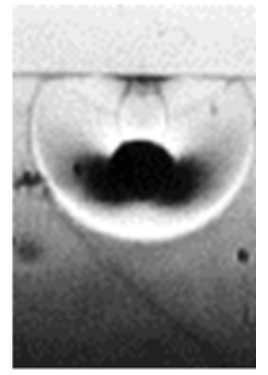

(e)

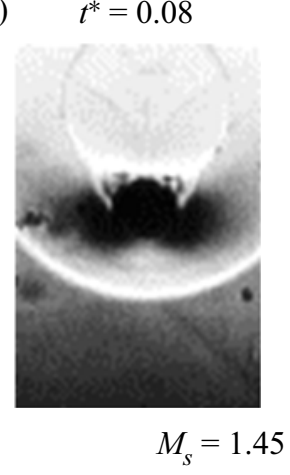

(f) $\quad t^{*}=0.36$

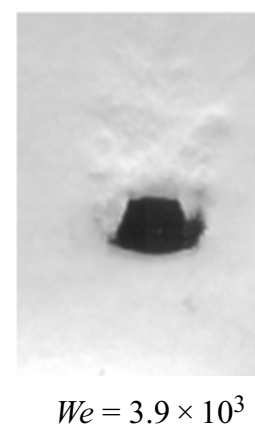

(g) $\quad t^{*}=0.44$

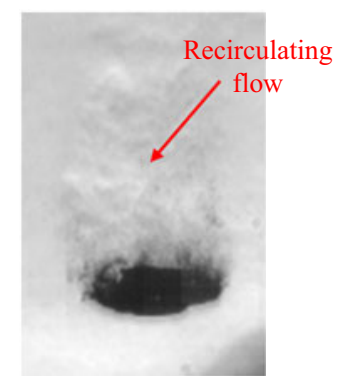

$\operatorname{Re}=6.5 \times 10^{4}$

Figure 5. Schlieren images recorded at $0.42 \mathrm{M}$ f.p.s. with $64 \times 96$ pixels resolution showing shock-droplet interaction mechanism. (a) Falling droplet approaches to the incident shock wave; $(b)$ formation of reflected wave; (c) Mach reflection; $(d)$ focusing of Mach waves at the leeward side of the droplet; $(e)$ generation of primary vortical structure near leeward side of droplet; $(f)$ droplet deformation and vortex stripping; and $(g)$ stripping of daughter droplets from the primary droplet periphery.

follows the incoming incident shock wave (see figure $4 h$ ). The formed reflected shock wave deflects the uniform airflow as it pass through it (figure $4 i-l)$. The induced flow remains subsonic in all the cases studied in this work; therefore, it gets deflected during its motion around the droplet surface without creating any other shock structures. The liquid motion inside the droplet and a high-pressure region near the droplet's front stagnation point prompted windward surface deformation of the droplet.

If the incident wave is a shock wave, the transmitted wave will always be a shock wave (Henderson 1989; Sembian et al. 2016; Guan et al. 2018; Das \& Udaykumar 2020) (figure $4 b$ ). The transmitted wave moves inside the droplet, resulting in a high-pressure region behind it and an induced fluid flow towards the leeward side of the droplet (figure $4 i$ ) (Guan et al. 2018). As the pressure waves can travel faster in water in comparison with air, the transmitted wave gets detached from the incident wave and reaches the droplet's leeward side at an earlier instant (figure 4c,j) (Sembian et al. 2016; Das \& Udaykumar 2020). On reaching the air-water interface at the leeward side, the transmitted wave reflects as an expansion wave $\left(Z_{\text {air }}<Z_{\text {water }}\right)$ (Henderson 1989) which focuses at a point inside droplet due to the concave profile of reflecting surface (figure $4 d$ ). Following focusing, the expansion wave propagates towards the windward side, where it is again reflected from the interface. This reflection of the transmitted wave from the windward and leeward side of the droplet continues with some energy loss after every collision until it completely decays. 


\section{S. Sharma, A.P. Singh, S.S. Rao, A. Kumar and S. Basu}

Diffraction of the Mach stem occurs after passing the equator point of the droplet. This contributes towards the lagging of the Mach wave near its contact point with the droplet surface (figure $4 e, k$ ). The Mach wave is formed axisymmetrically around the droplet surface and focuses at a point near the rear stagnation point (figures $4 f, l$ and $5 d$ ) which strengthens the shock and creates a local high-pressure region at that location. The internal flow direction remains unidirectional towards the leeward side of the droplet until the shock focuses at the droplet's rear stagnation point (figure $4 i-l$ ). A high-pressure region at the rear stagnation point deforms the droplet surface locally, triggering an upstream flow inside the droplet. The two counteracting flows, i.e. downstream flow from front stagnation point and upstream flow from rear stagnation point interact with each other at the local saddle point inside the droplet, which divert the two flows towards the droplet equator (figure $4 m, n$ ). During the development of external airflow around the droplet periphery, a toroidal vortex (primary vortex) is formed near the flow separation point (figure $4 m$ ), similar to the external flow around a solid sphere of similar Reynolds number. In later times, the primary vortex moves downstream and forms a recirculation flow region and effectuate a jet flow at the rear stagnation point. Two counter-rotating secondary vortices V2 and V3, that interact with the droplet surface are also formed between the flow separation point and the recirculating flow region. Subsequent to focusing at a point, Mach waves propagate on the leeward surface, thereby increasing the influence of the high-pressure region (figures $4 m$ and $5 d, e$ ). The combined effect of recirculated jet flow and spreading of the high-pressure region flattens the leeward side of the droplet and deforms it into a cupcake shape (figures $4 n$ and $5 f$ ). The observed wave dynamics of droplet interaction with incident shock wave is in accordance with the previous work on shock-water-column interaction (Igra \& Takayama 2003; Sembian et al. 2016) and various two-dimensional numerical simulations using spherical dispersions (Mehta et al. 2016; Sridharan et al. 2016; Guan et al. 2018; Das \& Udaykumar 2020), indicating the axisymmetric nature of the stage I interaction. The continuous deformation of the droplet increases the projected area for aerodynamic drag, which further enhances the flattening of the liquid droplet (figures $4 o$ and $5 g$ ) and leads to droplet breakup in later time instants. Figure 6 represents the various droplet breakup morphologies observed during its interaction with a shock wave of different strengths. Panels $(a, b)$ correspond to the bigger droplet size $\left(D_{i} \sim 2.5 \mathrm{~mm}\right)$ and panels $(c, d)$ correspond to smaller droplet size $\left(D_{i} \sim 0.5 \mathrm{~mm}\right)$. The left-hand side images correspond to the low Weber number while the right-hand side corresponds to the high Weber number. Figure 6 essentially explains that two types of breakup modes (i.e. SIE and RTP) are observed based on Weber number and droplet sizes, and the interaction becomes more vigorous as the Weber number increases. The details of these mechanisms are provided in following subsections.

\subsection{Global observation of shock-droplet interaction mechanism}

Figure 7 shows the global observation of the shock-droplet interaction mechanism. The complete interaction dynamics can be divided into two stages: initial shock interaction (stage I) and droplet breakup dynamics (stage II). As discussed earlier, the initial shock interaction corresponds to the formation of different wave structures and a local change of ambient conditions around the droplet. These changes result in droplet deformation; but the droplet retains its coherent structure during the interaction stage I. The droplet deformation begins due to high-pressure regions at the front and rear stagnation points (due to external airflow near the front stagnation point, recirculating flow and shock 
(a)

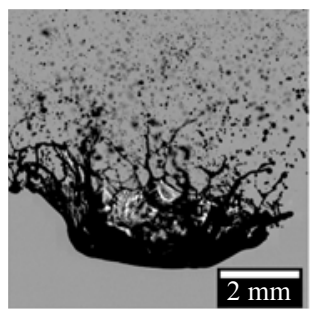

(c)

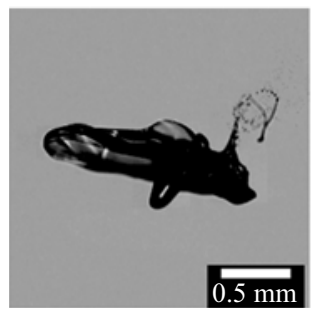

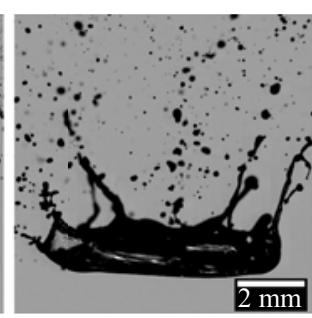

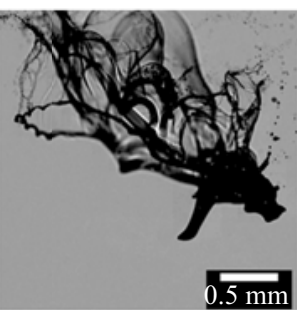

(b)
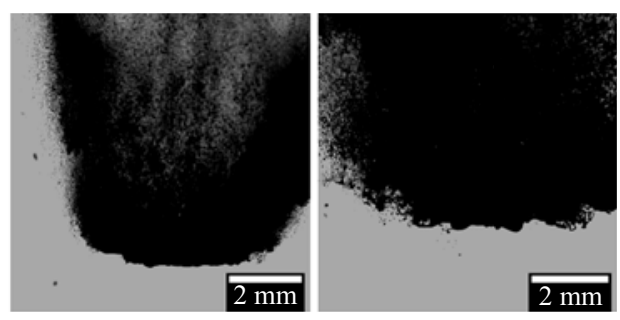

(d)
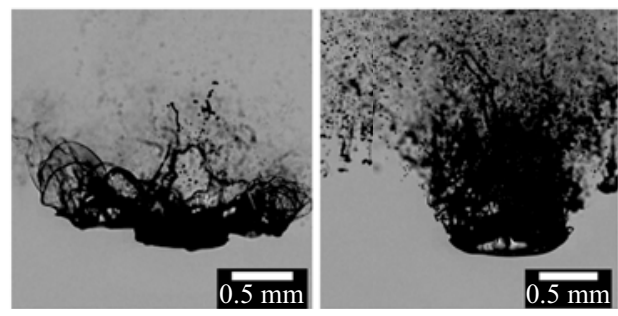

Figure 6. Droplet breakup morphologies for different shock Mach numbers and droplet sizes. (a) The SIE droplet breakup at $M_{s}=1.12, W e=219, R e=1.3 * 10^{4}, D_{i}=2.5$. (b) The SIE droplet breakup at $M_{s}=1.3$, $W e=1.6 * 10^{3}, R e=4 * 10^{4}, D_{i}=2.9$. (c) The RTP droplet breakup at $M_{s}=1.12, W e=44, R e=2.5 * 10^{3}$, $D_{i}=0.5$. (d) The RTP droplet breakup at $M_{s}=1.45, W e=795, \operatorname{Re}=1.2 * 10^{4}, D_{i}=0.5$.

focusing near the rear stagnation point), which overcomes the restoring surface tension and viscous forces acting on the droplet. A low-pressure region is formed near the equator of the droplet where the external flow velocity is comparatively higher, as in the case of external airflow over a cylinder/sphere of similar Reynolds number. Two high-pressure regions at the droplet's front and rear stagnation points and a low-pressure region near the equator direct the internal flow of liquid towards the droplet equator, resulting in the deformation of the spherical droplet into a cupcake shape. This pressure difference is further enhanced due to an increase in aerodynamic drag with deforming droplet shape. The shock-droplet interaction mechanism is universal up to this point for all cases of shock-droplet interaction. Beyond this (stage II interaction), depending on the relative dominance of pressure force or the shear force acting on the droplet surface, two modes of droplet breakup, i.e. RTP breakup (figure $7 b$ ) or SIE (figure 7c) breakup are observed.

The RTP-based droplet breakup is characterised by complete deformation of the droplet into a flattened disc followed by the formation of single/multiple bags, which grow over time due to incoming airflow and undergoes rupture. The bag rupture is followed by the breakup of the central undeformed region and outer toroidal rim joining the bags (Jackiw \& Ashgriz 2021). The shear-induced droplet breakup mode is characterised by the formation of KH-based surface waves (Theofanous et al. 2012; Guan et al. 2018; Dorschner et al. 2020) on the windward surface of the droplet. These waves undergo elongation and rupture, or the formed waves travel along the windward surface and merge to form a liquid sheet near the equator of the droplet. The external aerodynamic forces acting on the formed sheet lead to stretching in the cross-stream direction followed by rupture into fine mist (figure 7c). A detailed discussion on each droplet breakup mode is provided in the following subsections. 
Stage I: initial shock interaction

(a)

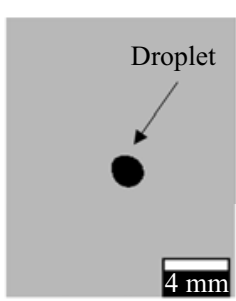

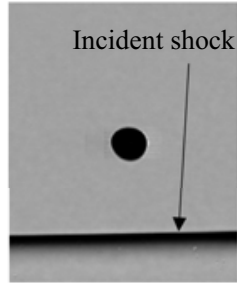

$M_{s}=1.16$ $t^{*}=0$

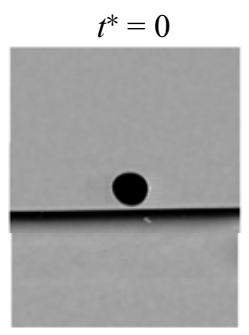

$W e=458$ $t^{*}=0.04$

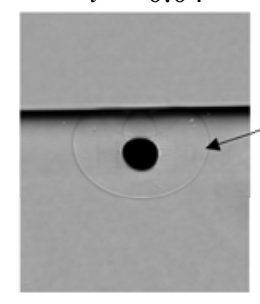

$R e=1.9 \times 10^{4}$ $t^{*}=0.08$

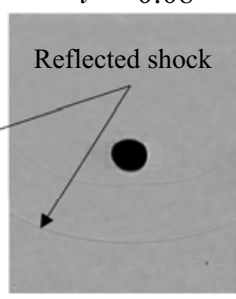

Stage II: droplet breakup dynamics

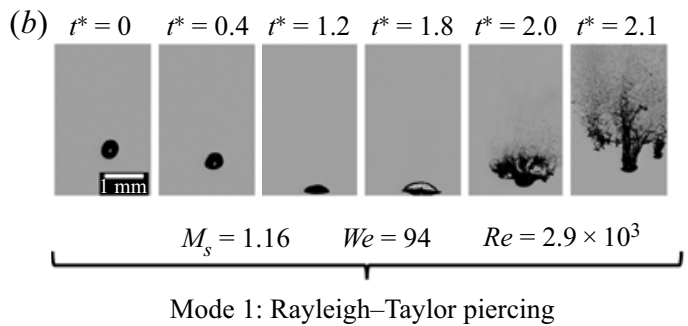

(c) $t^{*}=0 \quad t^{*}=0.21 \quad t^{*}=0.43 t^{*}=0.71 t^{*}=1.35$

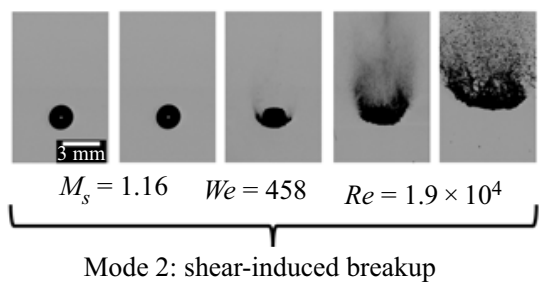

Figure 7. Global observation of shock-droplet interaction. (a) Stage I corresponding to the initial shock interaction; $(b)$ breakup dynamics (stage II) of a droplet $\left(D_{i} \sim 0.5 \mathrm{~mm}\right)$ undergoing breakup through RTP mode; and (c) SIE mode of droplet breakup for $D_{i} \sim 2.5 \mathrm{~mm}$.

\subsection{Shear-induced droplet breakup}

The initial stages of shock-droplet interaction involving wave dynamics and droplet deformation were discussed in $\S 3.1$. The present subsection strides towards understanding the breakup dynamics of a droplet through the SIE mode. Figure 8 (also supplementary movie 2) shows the time sequence images (from left to right) of the breakup dynamics of a $~ 2.9 \mathrm{~mm}$ diameter droplet for different values of shock Mach numbers. As discussed earlier, the initial wave interaction and droplet deformation stages are similar for all experimental cases. The deformed droplet attains a cupcake shape, after which it undergoes a stripping type of droplet breakup. The stripping of daughter droplets near the equator of the primary droplet is triggered by the formation of $\mathrm{KH}$ waves (Theofanous et al. 2012; Liu et al. 2018) near the front stagnation point of the droplet (see figure 8, third column). These waves are formed midway between the front stagnation point and droplet equator, along the droplet periphery, which is the region of highest shear (Liu et al. 2018). The validation of formed surface waves with theoretical predictions is shown later. For a better understanding of the breakup dynamics, a high-resolution (8 $\mu \mathrm{m} \mathrm{pixel}^{-1}$ ) and high-speed (at 50000 f.p.s.) imaging of interaction phenomena was done in a pendant drop mode (see figure 9). This configuration will affect the breakup dynamics at the rear stagnation point; however, no distinguishable difference between pendant mode and free-falling configuration could be identified at the windward side of the droplet (see supplementary movie 3). This methodology for obtaining high-resolution and high-speed imaging was also recently employed by Jackiw \& Ashgriz (2021). Figure 9(a,b) corresponds to the shock Mach number of 1.12 and 1.47, respectively. Once formed, $\mathrm{KH}$ waves grow in amplitude (figure $9 a t^{*}=0.37-0.4$ and figure $9 b t^{*}=0.1-0.29$ ) and subsequently get deflected towards the flow direction (figure $9 a t^{*}=0.47$ and 
(a)

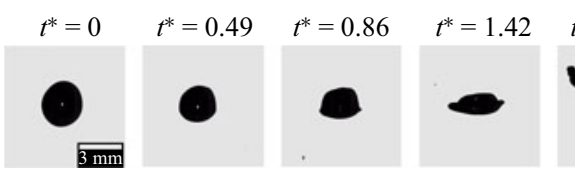

(b)

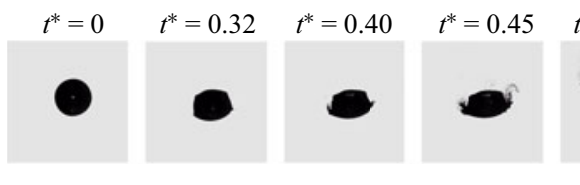

(c)

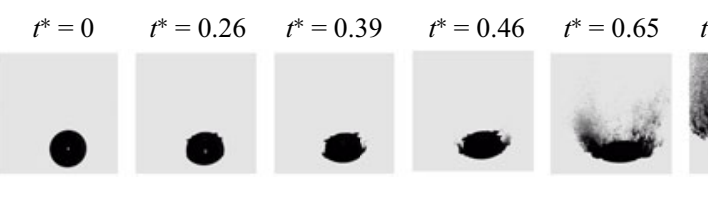

(d)

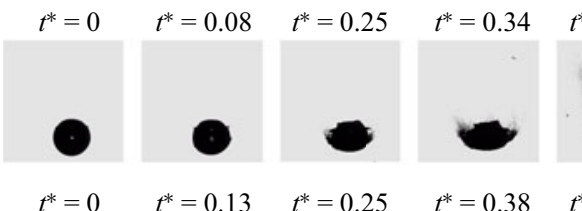

(e)

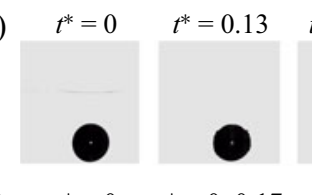

$(f)$
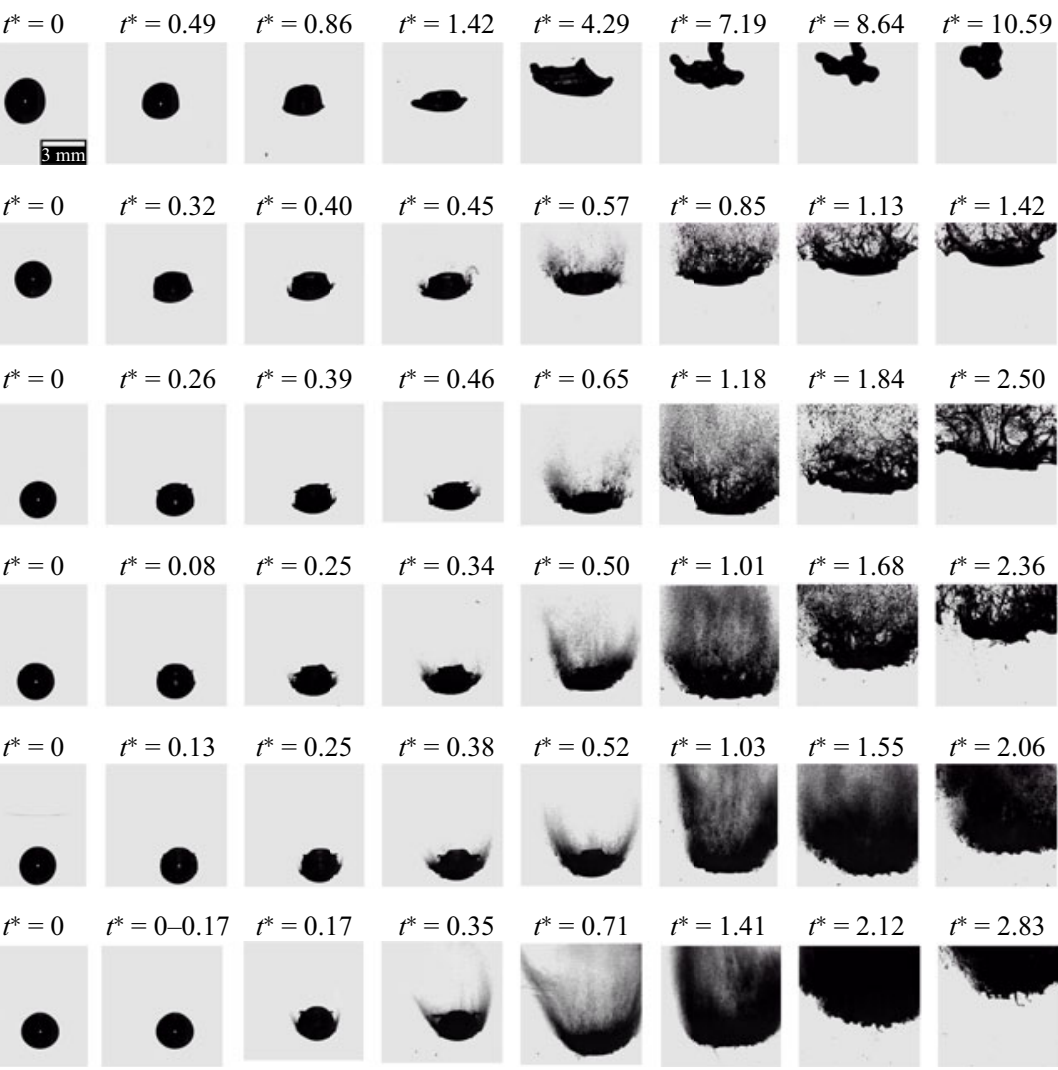

$M_{s}=1.17$
$W e=513$
$R e=2.2 \times 10^{4}$
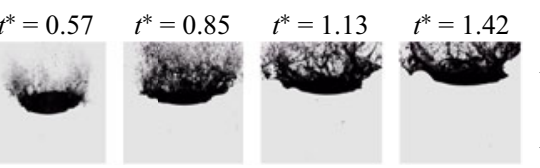

$M_{s}=1.27$

$W e=1.3 \times 10^{3}$

$R e=3.4 \times 10^{4}$

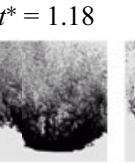

$t^{*}=1.84 \quad t^{*}=2.50$

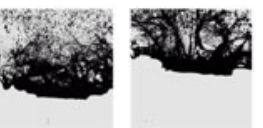

$M_{s}=1.35$

$W e=2.2 \times 10^{3}$

$R e=4.5 \times 10^{4}$

figure $9 b t^{*}=0.38$ ) when entrained by the external air flow (see figure 8 , fourth column). The flow entrainment causes the surface waves to move towards the downstream direction along the windward surface and after reaching the flow separation point (at the droplet equator) the liquid carried with these waves gets accumulated as a thin sheet at this location (figure $9 a t^{*}=0.47$ and figure $9 b t^{*}=0.38$ ). The thin sheet undergoes rupture due to nucleation of multiple holes (shown later through top-view imaging) which expands in size in a temporal fashion leading to sheet rupture into numerous cylindrical ligaments (figure $9 a t^{*}=0.52$ and figure $9 b t^{*}=0.48$ ). The formed cylindrical ligaments further undergo secondary atomisation into smaller droplets through Rayleigh-Plateau modes of instability.

In addition to the accumulation of droplet fluid near the equator due to $\mathrm{KH}$ waves (leading to sheet formation), the effect droplet deformation, which causes an internal flow directed towards the equator region (discussed in $\$ 3.1$ ) also augments the sheet formation at the equator (see figure $4 m-o$ ). Here, the fluid transport towards the equator through the droplet deformation is greater than micron-sized $\mathrm{KH}$ waves. Therefore, the sheet formation with the predominant $\mathrm{KH}$ wave mechanism will be thinner than the droplet deformation mechanism. Subsequently, the droplet sizes stripping from the rupture of the sheet will also be smaller for the KH-waves-based liquid accumulation mechanism. Therefore, during the 
(a)
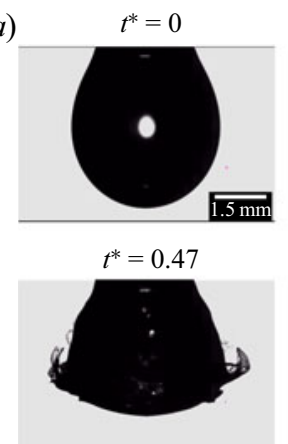

(b)
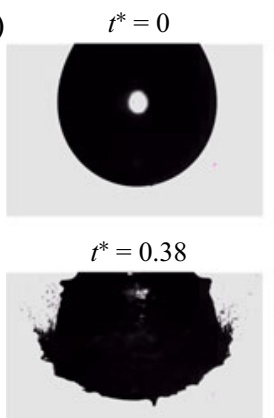

$t^{*}=0.37$
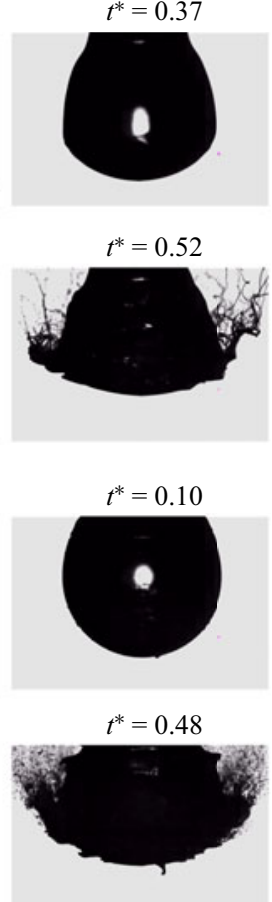
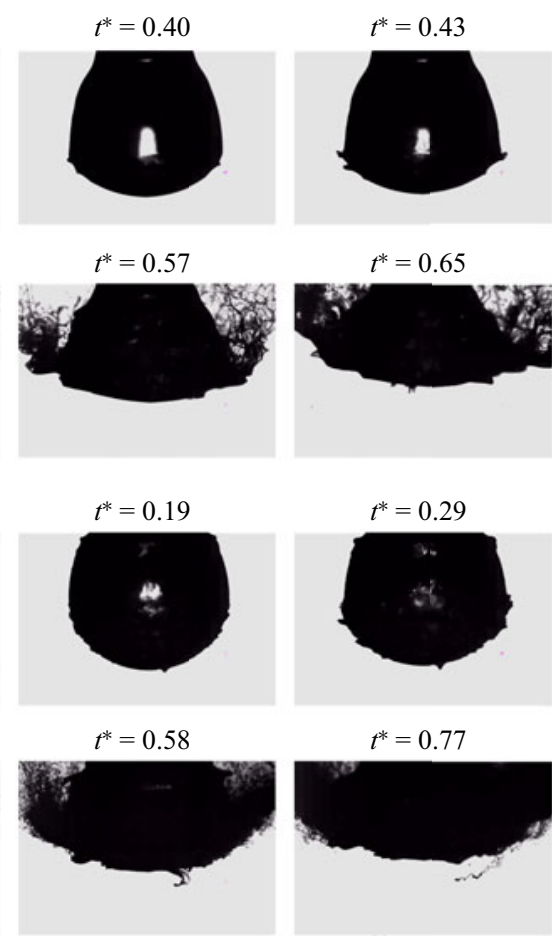

Figure 9. Zoomed-in view of shear-stripping breakup. (a) Time-sequence images of droplet breakup at $M_{s}=$ 1.12 and $D_{i}=2.02 \mathrm{~mm}$; and (b) time-sequence images of droplet breakup at $M_{s}=1.47$ and $D_{i}=2.24 \mathrm{~mm}$. For details see supplementary movie file 3 .

early stages of droplet breakup (see figure 8 , fourth and fifth columns), the extent of droplet deformation is less, and $\mathrm{KH}$ waves are the only dominant mechanism for sheet formation leading to a fine mist of daughter droplets.

Figures 8 and 9 and theoretical analysis shown later (\$3.4) shows that the number of $\mathrm{KH}$ waves formed on the droplet surface increases with increase in Weber number. Moreover, the local incubation location of $\mathrm{KH}$ waves shifts towards the front stagnation point as the Weber number increases (see figure 9). The formed surface waves grow in amplitude and move towards the equator of the droplet, where they get accumulated at the stripping sheet and compensate for the liquid mass loss due to sheet rupture. The thin sheet formed due to $\mathrm{KH}$ waves experiences two driving forces leading to its motion: first, the entrainment force due to the shear acting on its surface by external airflow; and second, additional pull due to the induced internal motion by the movement of $\mathrm{KH}$ wave formed ahead of it (towards the equator). Therefore, the translational velocity of the wave formed near the front stagnation point is higher than the one formed near the equator. This results in merging of two surface waves as they translate towards the droplet equator, resulting in an increase of combined wave sheet thickness. The merging of surface waves can be seen in figure $9(b)$ $\left(t^{*}=0.19-0.48\right)$ and is shown more clearly in next subsection. The merged KH waves on reaching the droplet equator result in the formation of a thicker sheet; thereby the size of fragmenting daughter droplet increases (see figure 8, fifth column). The thickness of the stripping sheet is further augmented by the deformation of the primary droplet, which again increases the size of fragmenting droplets. Therefore, the size of daughter droplets monotonically increases from the beginning of breakup to the end during the SIE breakup 


\section{Shock induced aerobreakup of a droplet}

regime (see figure 8). The breakup mechanism involving sheet formation at droplet equator due to the combined effect of accumulation of $\mathrm{KH}$ waves and droplet deformation occurs recurrently (discussed later) until the complete droplet disintegrates into daughter droplets or aerodynamic forces acting on the remaining sheet of the deformed droplet are unable to overcome the surface tension of the sheet. The stretched sheet in the latter case retracts back to form a single droplet.

More surface waves are formed at the higher Weber numbers (figure 8). This implies that the amount of liquid transported through these waves will also be higher. In addition to this, the extent of droplet deformation also decreases with increasing Weber number. Therefore, higher wavenumbers and lower droplet deformations result in the domination of KH-waves-based liquid transport mechanism at the stripping sheet, which therefore explains the formation of smaller droplet sizes with increasing shock Mach number (see figures 8 and 9). In contrast to this, at lower Mach numbers, the number of $\mathrm{KH}$ waves formed are lower, and the extent of droplet deformation is higher; therefore, the formed droplet sizes are bigger in such cases. The above explanation of SIE breakup based on the competing effect of $\mathrm{KH}$ waves and droplet-deformation-based liquid transmission towards the stripping sheet has been reported for the first time in this work. This explains both the temporal increase in the size of daughter droplets and reduction in daughter droplet sizes with increasing Weber number, observed during SIE-based breakup.

In addition to the surface waves formed at the windward surface of the droplet, a flattened disc and a lip are also formed on the leeward side of the droplet due to the internal flow induced inside the droplet by shock focusing and jet flow impinging on the rear stagnation point of the droplet (discussed in $\$ 3.1$ ). The formed lip interacts with the secondary vortices formed after external flow stabilisation around droplet (figure $4 n$ ). These formed vortices stretch and engulf the droplet lip sheet towards their core, as explained by Sharma, Singh \& Basu (2021), leading to its rupture. This mechanism also contributes to mist formation during SIE-based droplet breakup; however, it lasts for a short time interval and has an insignificant contribution in the total number of daughter droplet formations (figure 8).

\subsection{Kelvin-Helmholtz-based surface instabilities}

The previous subsection discusses the droplet breakup mechanism based on KH surface waves. Here, we present the theoretical analysis used to predict the wavelength of the formed surface waves. Existing models of $\mathrm{KH}$ instabilities based on zero and non-zero vorticity thickness (Marmottant \& Villermaux 2004; Jalaal \& Mehravaran 2014) are used for the comparison of theoretical and experimental results. Figure 10 (also supplementary movie file 4) shows a zoomed-in view of the droplet windward surface which display the time sequence images of $\mathrm{KH}$ waves formation for Weber number values of We $=2095$ (figure 10a) and $W e=91667$ (figure 10b), respectively. As can be seen from figure 10, instability waves are formed much before the droplet response time (i.e. the beginning of droplet deformation). As discussed earlier, after formation, these waves move towards the droplet equator and sometimes merge to form a combined wave, thereby increasing the thickness of the liquid sheet carried along with them. Figure 10 shows at $t^{*}=0.13-0.19$ and $t^{*}=0.46-0.52$, some KH-wave-induced liquid sheets are unable to sustain further elongation and undergo rupture before accumulating at the droplet equator. This is an additional mechanism responsible for mist formation during SIE-based droplet breakup.

Considering an inviscid and incompressible airflow on the droplet surface, no gravity effects and negligible vorticity layer thickness (see figure 10c), one can apply the first-order 
(a)

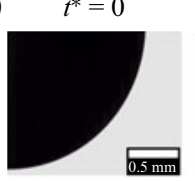

$t^{*}=0.52$

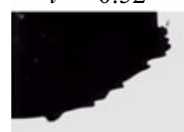

(b)

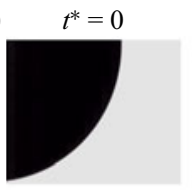

$$
t^{*}=0.27
$$
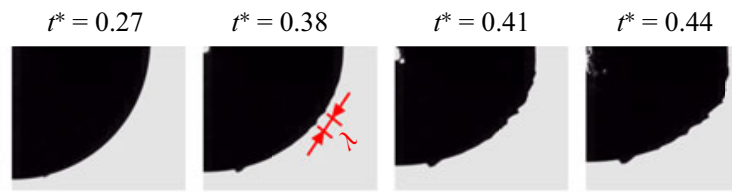

$t^{*}=0.58$
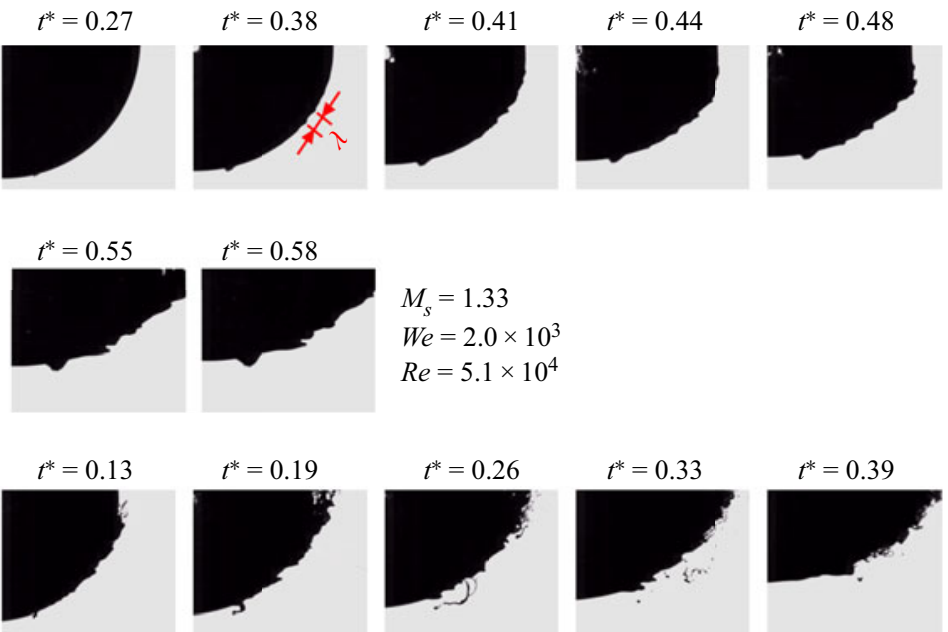
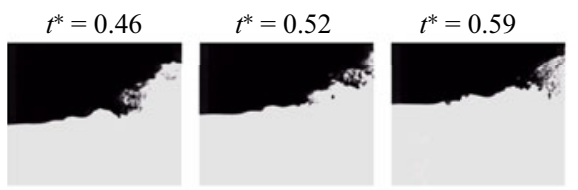

$M_{s}=1.60$
$W e=7.5 \times 10^{3}$
$R e=9.2 \times 10^{4}$

(c)

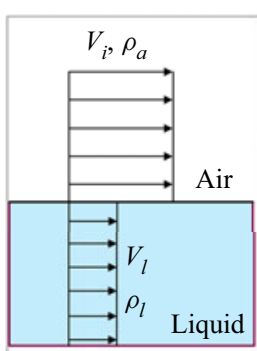

$(d)$

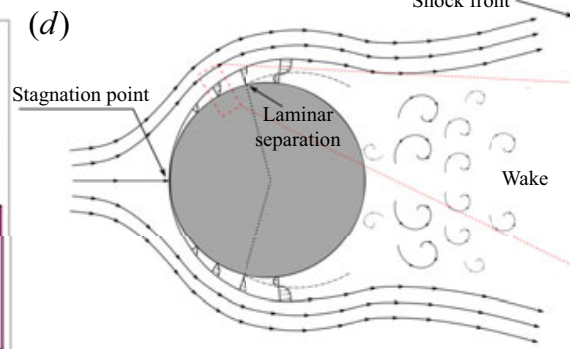

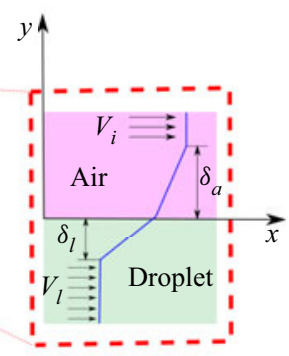

Figure 10. Kelvin-Helmholtz instability near front stagnation point of droplet for $D_{i} \sim 2.5 \mathrm{~mm}$. Time sequence images of $\mathrm{KH}$ waves formation is shown for $(a) M_{s} \sim 1.16$ and $(b) M_{s} \sim 1.45$ from left to right. For details see supplementary movie file 4 . Panels $(c)$ and $(d)$ show the schematic diagram for zero and non-zero vorticity thickness models for $\mathrm{KH}$ waves, respectively.

linear perturbation analysis to obtain the following dispersion equation (Marmottant \& Villermaux 2004) for the instability growth rate $\left(\omega_{K H}\right)$ :

$$
\omega_{K H}=k_{K H} \frac{\rho_{l} V_{l}+\rho_{a} V_{i}}{\rho_{l}+\rho_{a}} \pm i \frac{k_{K H}}{\rho_{a}+\rho_{l}} \sqrt{\rho_{l} \rho_{a}\left(V_{i}-V_{l}\right)^{2}-\left(\rho_{a}+\rho_{l}\right) \sigma k_{K H}} .
$$

Here, $k_{K H}$ is the wavenumber of the instability, $V_{l}$ is the velocity of the droplet fluid and subscript $K H$ refers to $\mathrm{KH}$-based instability. In (3.1), surface tension will stabilise all shear instabilities above critical wavenumber $\left(k_{K H_{c}}\right)$, where

$$
k_{K H_{c}}>\frac{\rho_{a} \rho_{l}}{\rho_{a}+\rho_{l}} \frac{\left(V_{i}-V_{l}\right)^{2}}{\sigma} .
$$


Now, considering $V_{i} \gg V_{l}$ and $\rho_{l} \gg \rho_{a}$, which is a valid assumption in our case, one can obtain the wavenumber $\left(k_{K H_{\max }}\right)$ corresponding to maximum growth rate $\left(\omega_{K H_{\max }}\right)$ as

$$
\begin{gathered}
k_{K H_{\max }}=\frac{2}{3} \frac{\rho_{l} V_{i}^{2}}{\sigma}=\frac{2}{3} \frac{W e}{D_{i}}, \\
\lambda_{K H}=\frac{2 \pi}{k_{K H_{\max }}}=\frac{3 \pi}{W e} D_{i} .
\end{gathered}
$$

Figure 11 shows the comparison of experimental and theoretical prediction of KH-wave wavelength corresponding to maximum growth rate with different Weber numbers. As predicted by $(3.3 a)$, the wavelength of maximum growth rate decreases with an increase in Weber number. Although the zero-vorticity thickness model predicts the correct trend of experimental data, the absolute values were found to be $O(10-100)$ less than the experimental observation (see table 1, third column). Similar under-prediction of wavelength while using the assumption of zero-vorticity layer thickness was also reported by other researchers (Marmottant \& Villermaux 2004; Kim et al. 2006; Jalaal \& Mehravaran 2014). The impractical assumption of zero vorticity layer thickness between the external airflow and internal liquid flow led to this under-prediction of the theoretical model. Therefore, a modified model with a finite thickness of vorticity layer and linear variation of velocity profile (see figure 10d) based on the work of Lord Rayleigh (1879) for two different phases in the vorticity layer were obtained by Villermaux (1998) as

$$
\lambda_{K H}=\frac{2 \pi}{0.8} F\left(\frac{\rho_{l}}{\rho_{a}}\right) \delta_{a}
$$

where, $\delta_{a}$ is the boundary layer thickness of air phase (see figure $10 d$ ) and

$$
F\left(\frac{\rho_{l}}{\rho_{a}}\right)=\frac{5}{6}-\frac{1}{6\left(\rho_{l} / \rho_{a}\right)}+\frac{\sqrt{5+13\left(\rho_{l} / \rho_{a}\right)-37\left(\rho_{l} / \rho_{a}\right)^{2}+27\left(\rho_{l} / \rho_{a}\right)^{3}}}{6 \sqrt{2}\left(\rho_{l} / \rho_{a}\right)} .
$$

The value of $\delta_{a}$ for the present work is obtained using boundary layer analysis, where

$$
\delta_{a} \sim \frac{L_{c}}{\sqrt{R e_{L_{c}}}}=C_{1} \frac{L_{c}}{\sqrt{\operatorname{Re}_{L_{c}}}} .
$$

Here, $L_{c}$ is the characteristic length scale, which in the present case is the initial drop radius $\left(R_{i}\right)$ and $C_{1}$ is constant replacing scaling analysis and its value is chosen as a fitting parameter for figure 11. For the limiting case of $\left(\rho_{l} / \rho_{a}\right) \gg 1$, (3.4) can be written as (Villermaux 1998)

$$
\lambda_{K H}=\frac{2 \pi}{1.5} \sqrt{\frac{\rho_{l}}{\rho_{a}}} \delta_{a},
$$

and the corresponding dispersion equation is obtained as (Marmottant \& Villermaux 2004)

$$
\mathrm{e}^{-2 \kappa}=\left[1-\left\{2 \Omega^{*}+\kappa\right\}\right] \frac{1+0.5\left(\rho_{l} / \rho_{a}+1\right)\left(2 \Omega^{*}-\kappa\right)}{1+0.5\left(\rho_{l} / \rho_{a}-1\right)\left(2 \Omega^{*}-\kappa\right)} .
$$

Here, $\kappa=k_{K H} \delta_{a}$ and $\Omega^{*}=\omega_{K H} \delta_{a} /\left(V_{i}-V_{l}\right)-2 \kappa\left(\left(V_{i}+V_{l}\right) /\left(V_{i}-V_{l}\right)\right)$. The ratio of predicted and experimental wavelength based on (3.7) and (3.4) is referred as 'non-zero thickness ratio 1' and 'non-zero thickness ratio 2' in table 1 and figure 11 . The decreasing trend of KH wavelength $\left(\lambda_{K H}\right)$ is well predicted by two models and in comparison with 


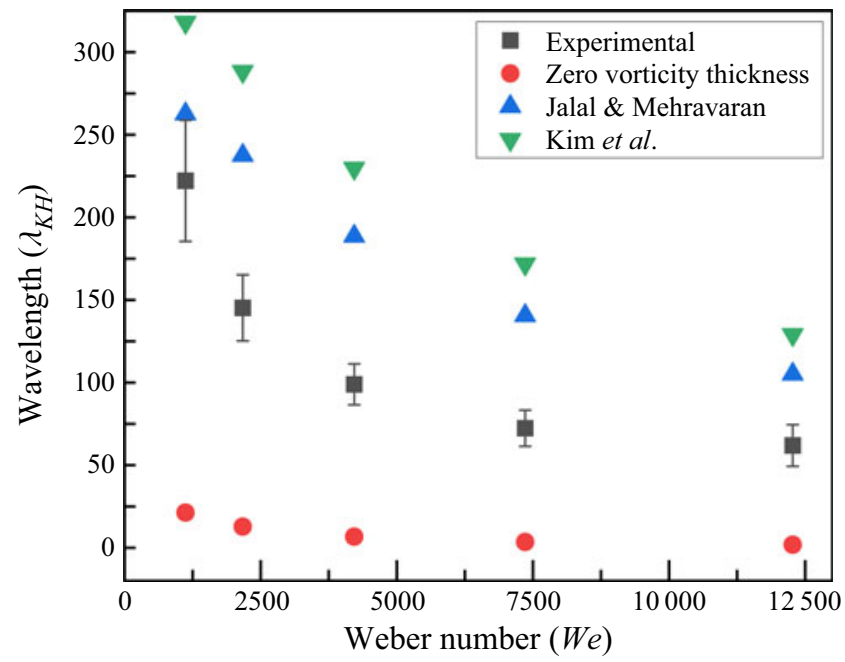

Figure 11. Wavelength variation of KH waves with shock Mach number $\left(M_{S}\right)$ for droplet size $\left(D_{i}\right)$ of $\sim 2.5 \mathrm{~mm}$.

\begin{tabular}{ccccc}
\hline & & $\begin{array}{c}\text { Zero thickness } \\
\text { ratio }\end{array}$ & $\begin{array}{c}\text { Non-zero thickness } \\
\text { ratio 1 }\end{array}$ & $\begin{array}{c}\text { Non-zero thickness } \\
\text { ratio } 2\end{array}$ \\
1.26 & $222.14 \pm 36.6$ & 0.10 & 1.10 & 1.33 \\
1.33 & $145.18 \pm 20.0$ & 0.09 & 1.53 & 1.85 \\
1.45 & $98.94 \pm 12.3$ & 0.07 & 1.78 & 2.17 \\
1.60 & $72.35 \pm 10.9$ & 0.05 & 1.94 & 2.37 \\
1.79 & $61.93 \pm 12.6$ & 0.03 & 1.70 & 2.08
\end{tabular}

Table 1. Kelvin-Helmholtz instability comparison with theoretical models.

zero thickness model the predicted results based on non-zero vorticity thickness are of same order of magnitude of experimental data. The observed discrepancy in the absolute value of wavelength was also reported by Marmottant \& Villermaux (2004), Kim et al. (2006) and Jalaal \& Mehravaran (2014), and is attributed to the linear velocity profile assumption and error in estimating $C_{1}$ in (3.6). Based on the experimental observations of the present work, no significant trend of $\mathrm{KH}$ wavelength variation along the droplet periphery could be identified, as the measured wavelengths were found to be distributed randomly. Qualitatively KH waves are generally formed at an azimuthal location midway between the front stagnation point and droplet equator (i.e. region of highest shear Liu et al. 2018). The region near the front stagnation point remains smooth/unaffected. However, as the Weber number increases, the starting location of $\mathrm{KH}$ instabilities shift towards the front stagnation point, as can be seen from figure 9 .

\subsection{Top-view visualisation and recurrent breakup of liquid sheet}

The high-speed shock-droplet interaction is essentially a three-dimensional phenomenon, which necessitates top-view imaging to acquire relatively more information. The time-sequence images showing the top view of droplet breakup dynamics is shown in figures $12(a)$ and $12(b)$ for Weber numbers values of 474 and 4133, respectively. 
(a)
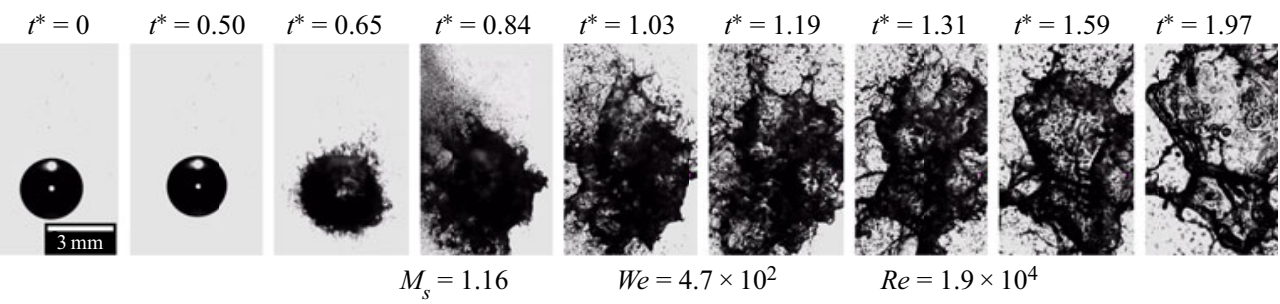

(b)
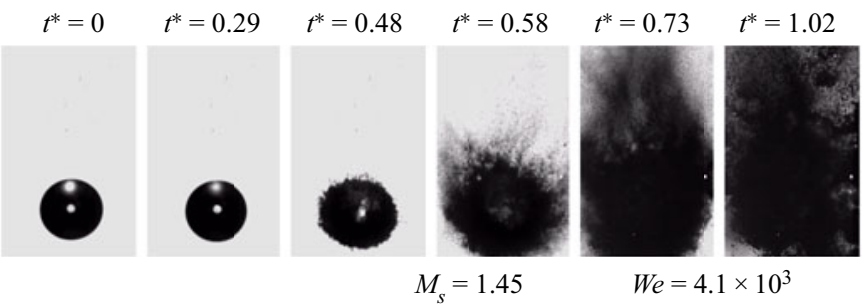

$W e=4.1 \times 10^{3}$

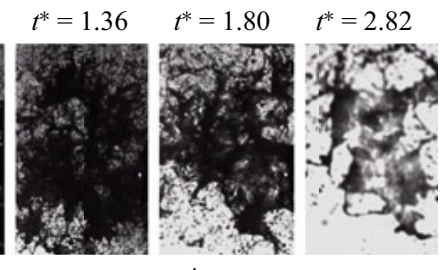

Figure 12. Time sequence images showing the top view of shock-droplet interaction for droplet size $\left(D_{i}\right)$ of $\sim 2.5 \mathrm{~mm}$ at $(a) M_{s} \sim 1.3$ and $(b) M_{s} \sim 1.46$.

As discussed earlier, the breakup dynamics are similar for both cases. However, the fragmentation of the primary droplet is more intense at a higher Weber number, leading to much finer daughter droplet sizes after the breakup. The ligaments at the droplet periphery are formed periodically along the azimuthal direction during the first breakup (figure $12 a$ at $t^{*}=0.65$ and figure $12 b$ at $t^{*}=0.48$ ). The periodic formation of fragmenting ligaments can be attributed to azimuthal modulation of the RT waves on the existing KH waves formed due to shear effects. In continuation of the work of Villermaux (1998) and Marmottant \& Villermaux (2004) involving instability analysis of a liquid jet exposed to a coaxial airflow, Jalaal \& Mehravaran (2014) carried a similar analysis on a droplet exposed to external uniform flow and explained RTI-based azimuthal modulations on axisymmetric $\mathrm{KH}$ waves (formed due to shear) due to the acceleration of $\mathrm{KH}$ waves towards the droplet equator. The modulation of RT waves on the KH waves is shown in figure 13. Here figure 13(a) shows the cross-sectional location on which RT waves are shown in figure 13(b). The crest of RT waves get entrained to external airflow and result in ligament formation. The measurement of the RT wavelength was performed by measuring the distance between the stripping ligaments (as shown in figure 13c). An assumption is made that the formed RT waves stretch to form ligaments whose breakup can be seen through top-view imaging, as shown in the third column image of figures 12(a) and 12(b). The measurements are provided for a possible estimation in terms of order of magnitude basis. The exact values of RT wavelength could not be identified based on these images as randomness arises in the measurements due to the uncertain manner of ligament breakup under the influence of induced airflow. Therefore, values provided in table 2 must only be used for order-of-magnitude comparison. Similarly, no trend in the variation of these wavelengths could be explained with a change in shock Mach numbers using table 2.

A comparison of the experimentally observed RT-waves wavelength is made with an existing model based on Marmottant \& Villermaux (2004) analysis as

$$
\lambda_{R T} \cong 2.8 \delta_{a} W e_{\delta_{a}}-1 / 3\left(\frac{\rho_{a}}{\rho_{l}}\right)^{-1 / 3} .
$$


(a)

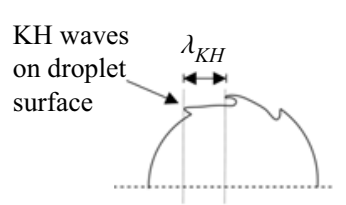

(b)

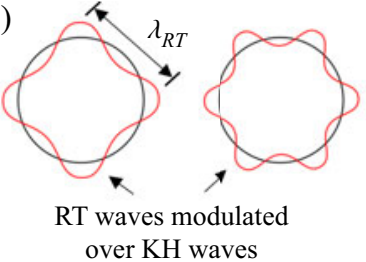

(c)

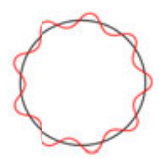

Increasing direction of $W e$

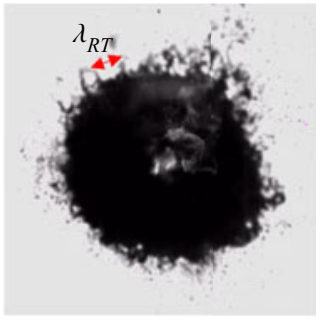

Top view

Figure 13. Azimuthal modulation of RT waves on KH waves. (a) Kelvin-Helmholtz waves formed near the front stagnation point of the droplet. Dashed lines show the cross-section of the KH wave on which RT waves are displayed. (b) Schematic diagram showing RT waves formed on droplet surface and their wavelength variation $\left(\lambda_{T}\right)$ with Weber number $(W e)$. (c) Experimental image showing RT waves leading to droplet stripping during initial stages of droplet breakup.

\begin{tabular}{|c|c|c|c|}
\hline$M_{s}$ & $\begin{array}{c}\text { Experimental } \\
\text { wavelength }\left(\lambda_{T_{\text {exp }}}\right) \\
(\mathrm{mm})\end{array}$ & $\begin{array}{c}\text { Theoretical } \\
\text { wavelength }\left(\lambda_{T_{\text {model }}}\right) \\
(\mathrm{mm})\end{array}$ & $\lambda_{T_{\text {exp }} / \lambda_{T_{\text {model }}}}$ \\
\hline 1.12 & $0.52 \pm 0.20$ & 1.86 & 3.57 \\
\hline 1.16 & $0.42 \pm 0.15$ & 1.15 & 2.75 \\
\hline 1.30 & $0.57 \pm 0.23$ & 0.59 & 1.02 \\
\hline 1.45 & $0.49 \pm 0.16$ & 0.32 & 0.66 \\
\hline
\end{tabular}

Table 2. Rayleigh-Taylor modulation over KH waves. A comparison of experimental and theoretical wavelengths.

Here, $W e_{\delta_{a}}$ is a Weber number based on the vorticity layer thickness. The comparison of the theoretical prediction with experimental data is shown in table 2. Although the predicted results correlate with experimental data with the same order of magnitude, the absolute magnitudes of predicted wavelengths differ considerably (up to three times). The observed discrepancy could be due to the linear velocity profile assumption between the vorticity thickness layer and constant $C_{1}$, as discussed earlier, and similar discrepancies were also obtained in the numerical simulations of Jalaal \& Mehravaran (2014). The RT waves measurements were only done for the first instance of droplet breakup, corresponding to the $\mathrm{KH}$ waves formed nearest to the droplet equator. This limitation was due to merging $\mathrm{KH}$ waves and additional ligaments formation due to sheet rupture during later breakup instances.

Jarrahbashi \& Sirignano (2014) had studied through vorticity dynamics the instability mechanisms in round liquid jets. The azimuthal instability on the axisymmetric $\mathrm{KH}$ waves can arise through the baroclinic effect (RTI, as shown above and in Marmottant \& Villermaux (2004)) and/or by streamwise vortex generation due to vortex tilting and stretching mechanisms (as in isodensity jets (Liepmann \& Gharib 1992)) leading to lobe formation. The baroclinic effects are dominant during atmospheric gas pressures (i.e. at low gas-liquid density ratios), where the gas-liquid density ratio is $O\left(10^{-3}\right)$. In such cases, RT instability is a dominant mode for lobe generation due to the high-density gradient across the interface (related to baroclinic vorticity). Also, the contribution from the vortex tilting and stretching mechanism is negligible because of lower gas inertia, thereby lower vortex strain near the liquid interface. However, at higher gas-density ratios, 
vortex-strain interactions are substantial and the contribution of the baroclinic effect decreases in streamwise vorticity generation. Therefore, stretching and tilting of vortices is a dominant mechanism in azimuthal modulation on KH waves for such cases (Jarrahbashi et al. 2016). A similar observation was also obtained for a liquid sheet exposed to external airflows (Zandian, Sirignano \& Hussain 2017, 2019a,b). For the shock Mach number cases considered in the present work, the gas-liquid density ratio is $O\left(10^{-3}\right)$, and therefore the baroclinic effect (or RTI) mode of lobe generation will be predominant, as discussed earlier.

Further, formed lobes undergo a cascade process through three different mechanisms during ligaments/droplet formation. The involved mechanisms include: (i) lobe stretching leading to ligament formation; (ii) corrugation on the lobes leading to multiple ligament formations during stretching of corrugation; and (iii) perforation of holes on the lobe leading to bridge breakup and ligament formation (Zandian et al. 2017, 2019b). The mode of ligament formation was shown to be dependent on the modified Ohnesorge number $\left(O h_{m}=\sqrt{\left(W e_{g}\right)} / R e_{l}\right)$ and Reynolds number $\left(R e_{l}\right)$ magnitude (Zandian et al. 2017). In the present work, we have refrained from determining the exact mechanism of ligament formation as the time sequence evolution of ligament formation was beyond the spatial and temporal resolutions. The ligament formation is also obstructed during the top-view imaging. However, future experimental or numerical works are motivated to uncover the prevalent mechanism of ligament formation during lobe disintegration. The elongation of the lobes leading to ligament formation was shown to be a predominant mechanism during low gas-liquid density ratio by Jarrahbashi \& Sirignano (2014). Therefore, the same can be assumed to be prevalent in the present case while determining the wavelength of RT waves.

The phenomenon of SIE breakup of a droplet is recurrent, as shown in figure 14 and explained schematically using figure $14(f)$. Each breakup cycle involves the formation of a thin sheet at the droplet periphery. The elongation of the sheet due to its entrainment into the external airflow further decreases the sheet thickness, making it susceptible to surface instabilities. Any small perturbation on this sheet can result in the nucleation of holes on its surface (see figure 14a). After formation, these holes expand in all directions, leading to the sheet's rupture into cylindrical ligaments. The formed ligament further undergoes a secondary atomisation into a daughter droplet through RPI and, thus, completes one breakup cycle. The second cycles begin with the formation of the thin sheet due to the accumulation of liquid at the droplet periphery due to the combined effect of $\mathrm{KH}$ waves translation and droplet deformation (as explained earlier) and follow a similar breakup pattern as discussed above. The three cycles of SIE-based recurrent breakup of the droplet are shown in figure 14. A similar recurrent breakup of the liquid droplet was also shown by Dorschner et al. (2020) through numerical simulations.

\subsection{A shift in droplet breakup dynamics during transition regime $(100<W e<1000)$}

Previously, the droplet breakup dynamics of low viscosity Newtonian fluids $(O h<$ 0.1 ) were subdivided into five major regimes (i.e. vibrational breakup (We $<11$ ), bag breakup $(11<W e<35)$, multimode breakup $(35<W e<80)$, sheet thinning breakup $(80<W e<350)$ and catastrophic breakup $(W e>350))$ based on Weber number range (Guildenbecher et al. 2009). These regimes were reclassified by Theofanous \& Li (2008) based on hydrodynamics instabilities, where previously identified bag and multibag breakup modes were included in the RTP regime (based on RTI), while sheet thinning and catastrophic regimes correspond to the SIE mechanism (based on KHI). The RTP 


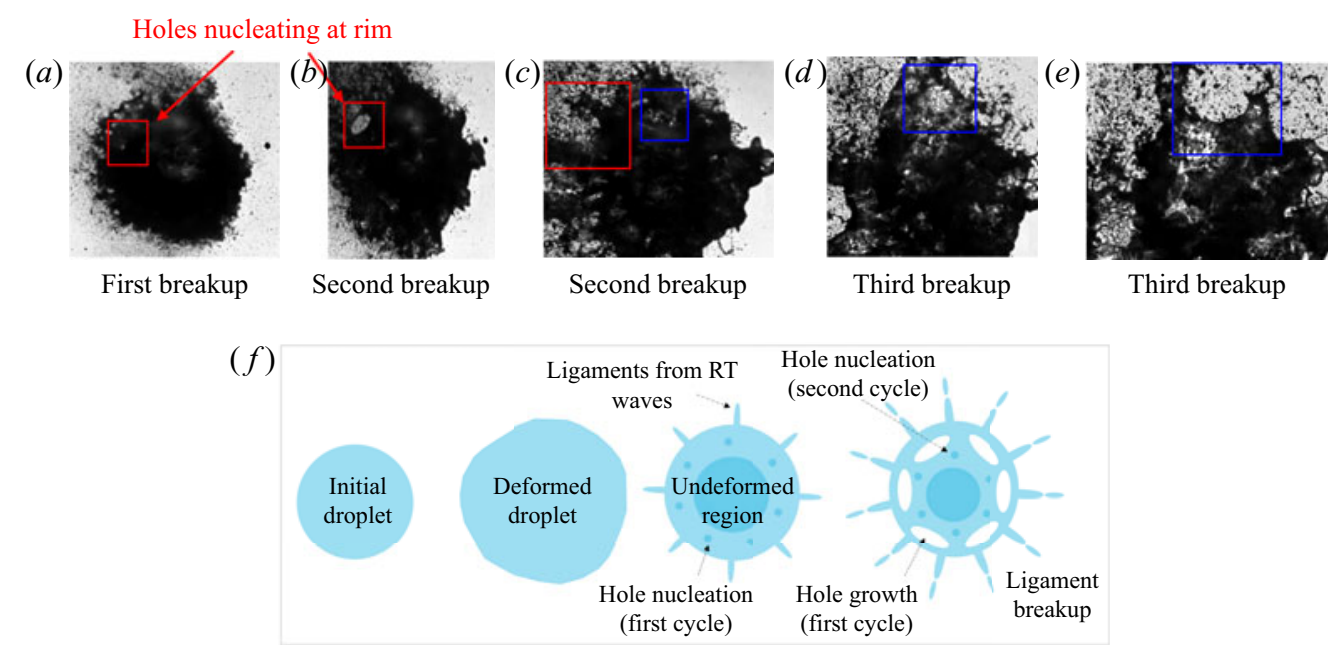

Figure 14. Recurrent breakup of the liquid sheet: $(a)$ first breakup; $(b, c)$ second breakup; and $(c-e)$ third breakup. The red and blue rectangular regions shows the time-sequence evolution of holes during second and third breakup, respectively. All images are of same scale. $(f)$ Schematic diagram showing recurrent breakup of a droplet.

regime ranges up to $W e<100$, the SIE regime corresponds to $W e>1000$, and a transition regime exists between these two regimes $(100<W e<1000)$. This reclassification is also validated in our experiments for RTP (figure 18a,b) and SIE (figure $8 b-f$ ) regime. In the transition regime, through figure 15 (also see supplementary movie files 5 and 6) we explain the dependence of the breakup mode on the size of the primary droplet. In the existing literature, a unidirectional breakup trend from bag breakup to catastrophic breakup is reported with increasing Weber number. The sheet thinning or catastrophic breakup are the only two breakup modes observed for We $>80$. Here, using the ratio of initial drop diameter to the wavelength of $\mathrm{KH}$ wave, we explain the existence of multimode droplet breakup for Weber number values up to $\sim 795$. Figure $15(a, b)$ shows the shear stripping mechanism, while figure $15(c, d)$ shows the multimode droplet breakup at a comparatively higher Weber numbers (We 347 795) values predicted from the previous studies $(W e<80)$. The important features in the evolution of multibag and shear stripping mode of droplet breakup are shown schematically in figure 15(e). The shear stripping breakup was discussed in detail in $\S 3.3$ and multibag breakup will be discussed later in this section. The growth rate of $\mathrm{KH}$ waves and RT waves on a droplet surface can be obtained from (3.8) and (3.10), respectively. The results of instability growth rate from dispersion relation based on $\mathrm{KH}$ wave (3.8) and $\mathrm{RT}$ wave (discussed later) are plotted in figure 16 for two particular cases of low and high Weber numbers. It is clearly observed that the growth rate of $\mathrm{KH}$ waves is always higher than the RT waves of corresponding Weber number, indicating that the RT waves (which lead to a bag-type breakup mechanism) based droplet breakup can only occur during the inability of $\mathrm{KH}$ wave formation (Theofanous et al. 2012). Thus, finding the criterion for this transition becomes important.

Figure 17 shows the ratio of droplet diameter with the predicted wavelength of $\mathrm{KH}$ wave based on (3.7). Green markers indicate the droplet sizes undergoing SIE-based breakup mode, while the red markers correspond to the RTP-based droplet breakup mechanism. It can be seen from figure 16 that $\mathrm{KH}$ waves are only formed if $D_{i} \gg \lambda_{K H}$ (at least by an order difference) while the RTP-based droplet breakup mode occurs when both $D_{i}$ 
(a)

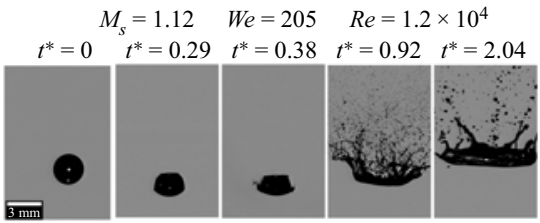

(b)

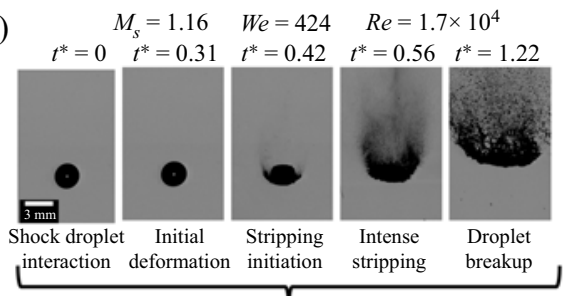

$D_{i} \sim 2.5 \mathrm{~mm}$ (c)

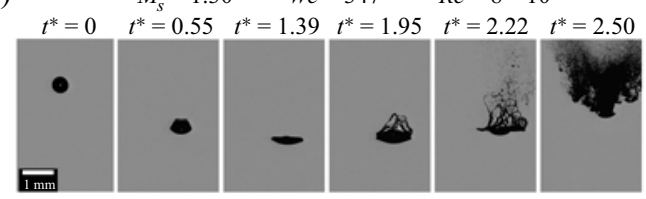

(d)

$M_{s}=1.45 \quad W e=795 \quad \operatorname{Re}=1.2 \times 10^{4}$

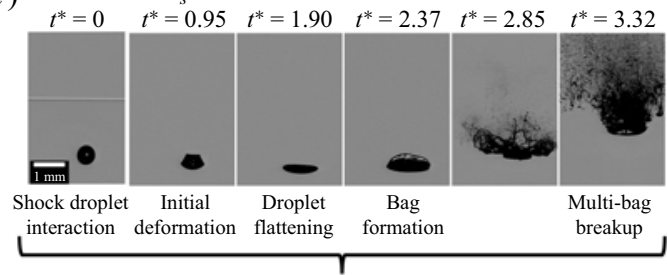

$D_{i} \sim 0.5 \mathrm{~mm}$

(e)
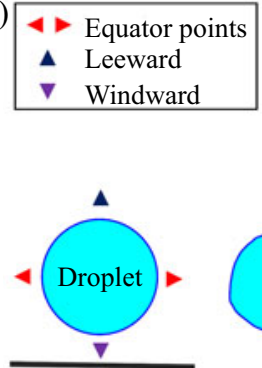

Shock wave

Shock interaction

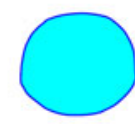

Initial deformation
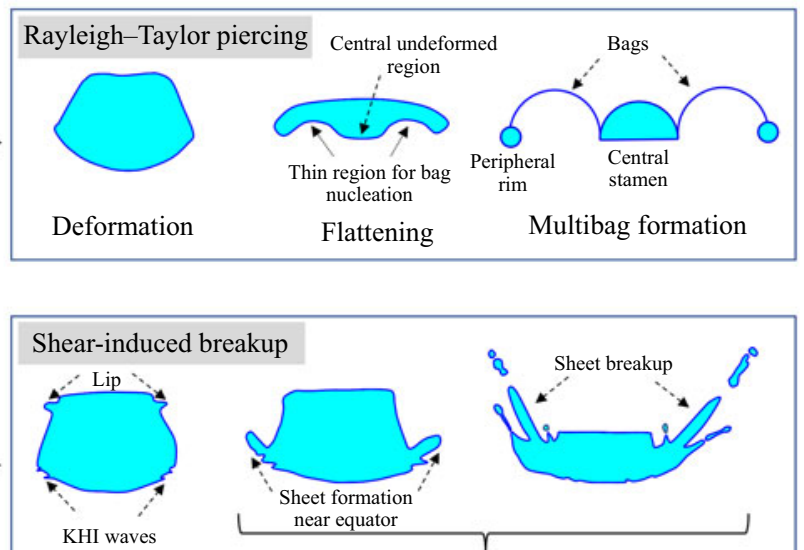

Surface waves
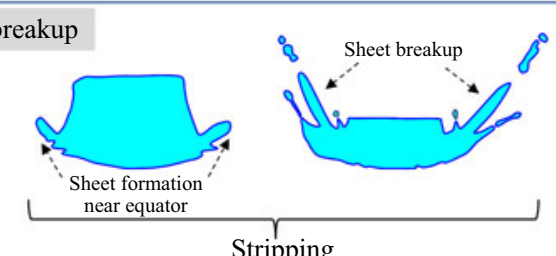

Figure 15. A shift of droplet breakup mechanism from SIE to RTP with different droplet sizes and similar Weber numbers $(W e)$. $(a, b)$ Time sequence images showing SIE mode of droplet breakup for $D_{i} \sim 2.5 \mathrm{~mm}$. $(c, d)$ Time sequence images showing RTP mode of droplet breakup for $D_{i} \sim 0.5 \mathrm{~mm}$. For details see supplementary movie files 5 and 6. (e) Schematic diagram showing droplet breakup through RTP and SIE modes.

and $\lambda_{K H}$ are of a similar order of magnitude. When $D_{i} \sim \lambda_{K H}$ the size of the fastest growing wavelength is similar or less than the size of the available area required for its formation, i.e. droplet windward surface length, which is of the order of the droplet radius. Therefore, KH waves cannot be formed under such cases (as predicted in figure 17), which gives way to the formation of much slower RT waves on the windward surface of the droplet after its complete deformation into a flattened disc. This explains the observation of multimode droplet breakup at Weber number values of $\sim 347$ and $\sim 795$. Thus, the present article indicates the importance of the $D_{i} / \lambda_{K H}$ ratio in addition to the Weber number for predicting the breakup mode of the droplet.

Figure 18 shows the RTP-based droplet breakup mechanism (also see supplementary movie file 7). In these cases, the primary droplet undergoes complete deformation (unlike SIE) into a flattened disc. The formed disc becomes unstable due to the acceleration of low density induced airflow on its surface, resulting in RTI. The growth rate $\left(\omega_{R T}\right)$ of RT 


\section{S. Sharma, A.P. Singh, S.S. Rao, A. Kumar and S. Basu}

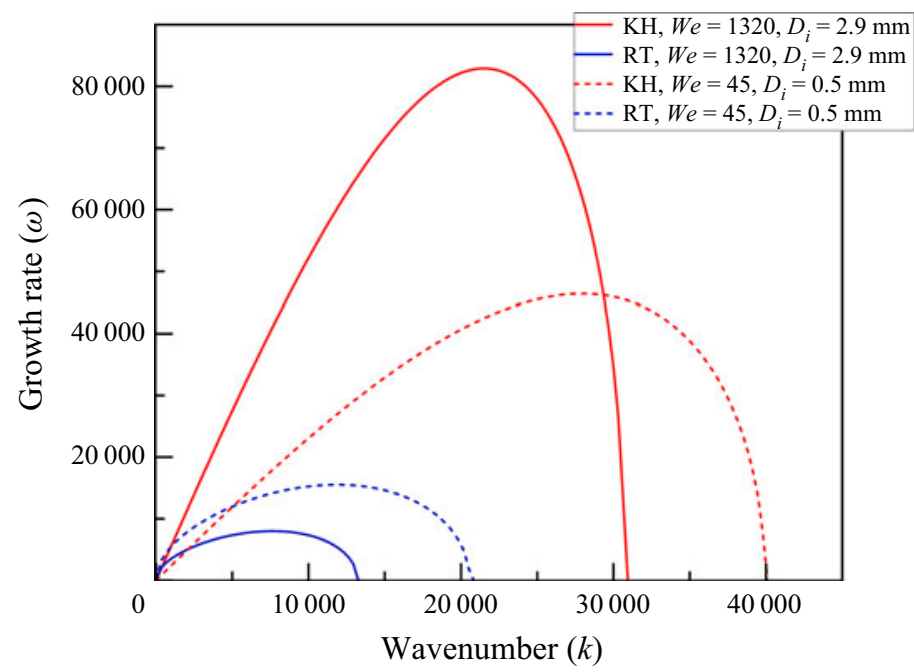

Figure 16. Rayleigh-Taylor and KH instabilities growth rate $(\omega)$ versus wavenumber $(k)$.

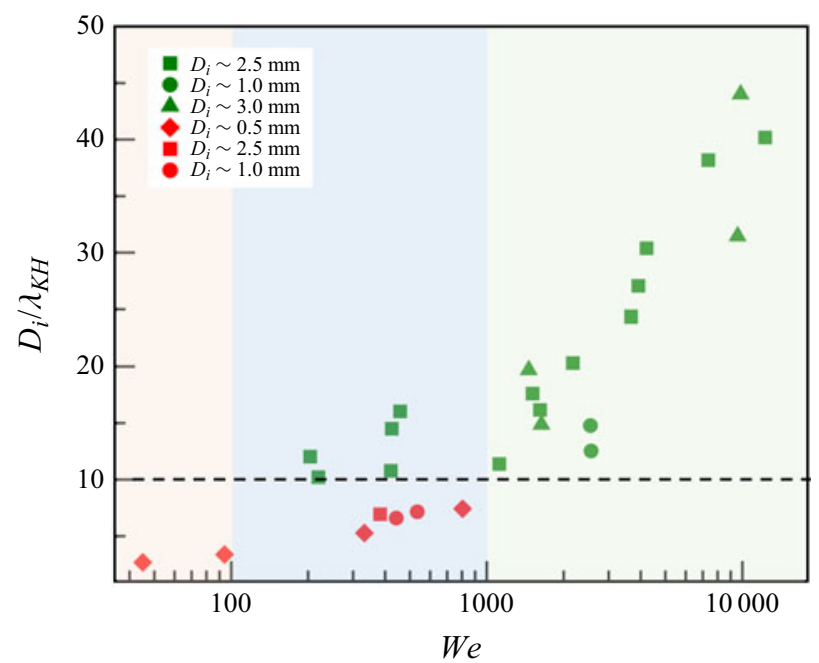

Figure 17. Transition of droplet breakup mode based on $D_{i} / \lambda_{K H}$ and We. Red markers indicates breakup thorough RTP mode while green markers correspond to the SIE mode. The orange region corresponds to RTP $(W e<100)$, the green region corresponds to SIE $(W e>1000)$ and the blue region corresponds to transition regime $(100<W e<1000)$.

instability can be expressed as (Drazin \& Reid 2004; Zhao et al. 2018)

$$
\omega_{R T}^{2}=\frac{k_{R T}\left[\left(\rho_{l}-\rho_{a}\right) a-k_{R T}^{2} \sigma\right]}{\rho_{l}+\rho_{a}} .
$$

Here, $k_{R T}$ is the wavenumber of RT waves, $a$ is the acceleration of the droplet, which is defined as

$$
a=\frac{F_{a}}{m_{d}}=\frac{3 \rho_{a} V_{i}^{2} C_{d} D_{m}^{2}}{4 D_{i}^{3} \rho_{l}} .
$$


(a)

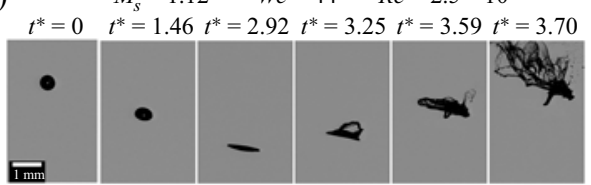

(b)

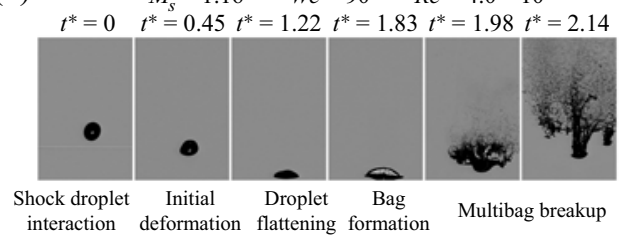

(c)

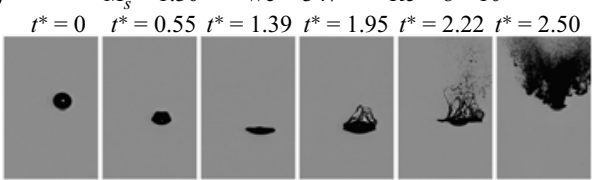

(d)

$M_{s}=1.45 \quad W e=795 \quad R e=1.2 \times 10^{4}$

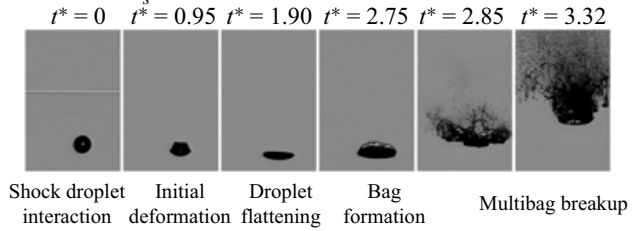

Figure 18. Droplet breakup dynamics at different Weber numbers for a droplet of diameter $\left(D_{i}\right) \sim 0.4 \mathrm{~mm}$. Time sequence images of droplet breakup from the instant of wire explosion to droplet breakup (from left to right). $(a-d)$ The magnitude of non-dimensional numbers $\left(M_{s}, W e, R e\right)$ increases from panels $(a)$ to $(d)$. For details see supplementary movie file 7 .

(a)
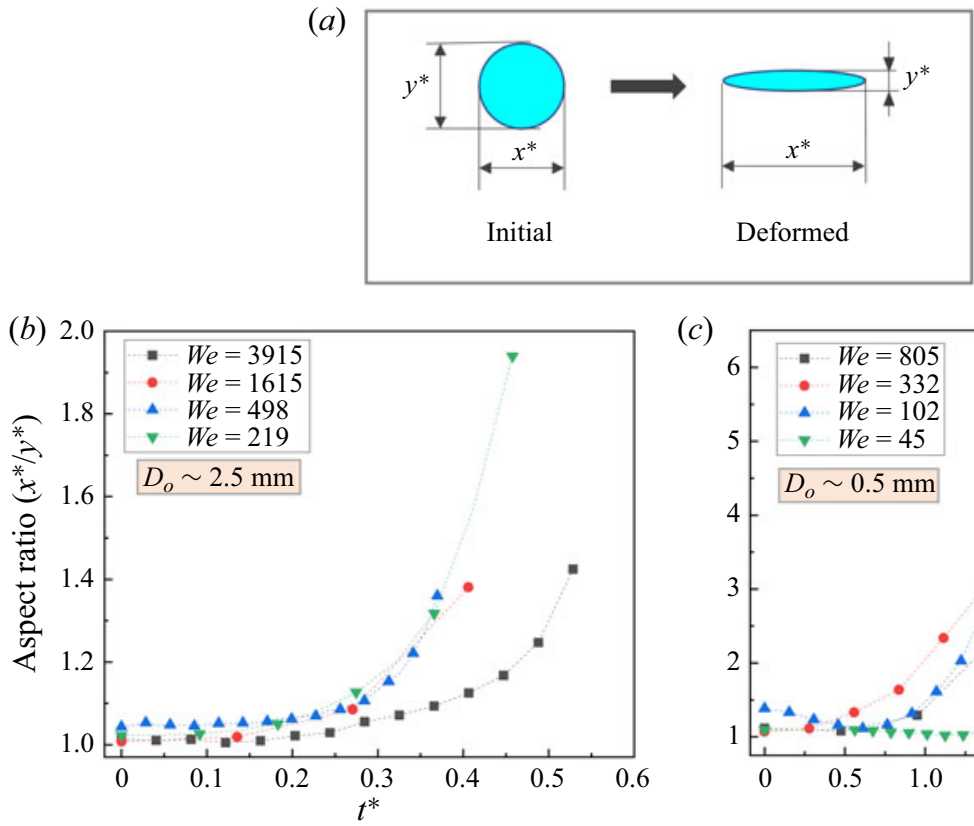

(c)

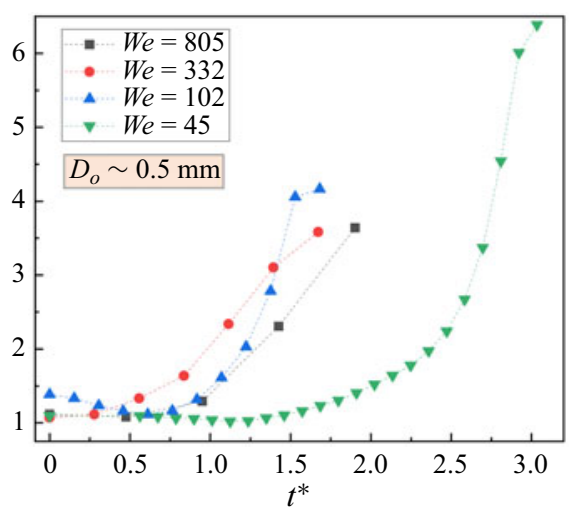

Figure 19. Aspect ratio variation with time: $(a)$ schematic diagram; and $(b, c)$ aspect ratio variation with time for droplet size of $\sim 2.5 \mathrm{~mm}$ and $\sim 0.5 \mathrm{~mm}$, respectively.

Here, $C_{d}$ is the drag coefficient whose value is chosen as $\sim 1.2$ (Zhao et al. 2018) and $D_{m}$ is the maximum droplet diameter during flattened state before the bag formation. The variation of RT instability growth rate for different wavenumber is shown in figure 16 . The wavelength corresponding to the maximum growth rate $\left(\lambda_{R T}\right)$ is obtained as (Drazin \& Reid 2004; Jain et al. 2015)

$$
\lambda_{R T}=2 \pi \sqrt{\frac{3 \sigma}{\rho_{l} a}} .
$$




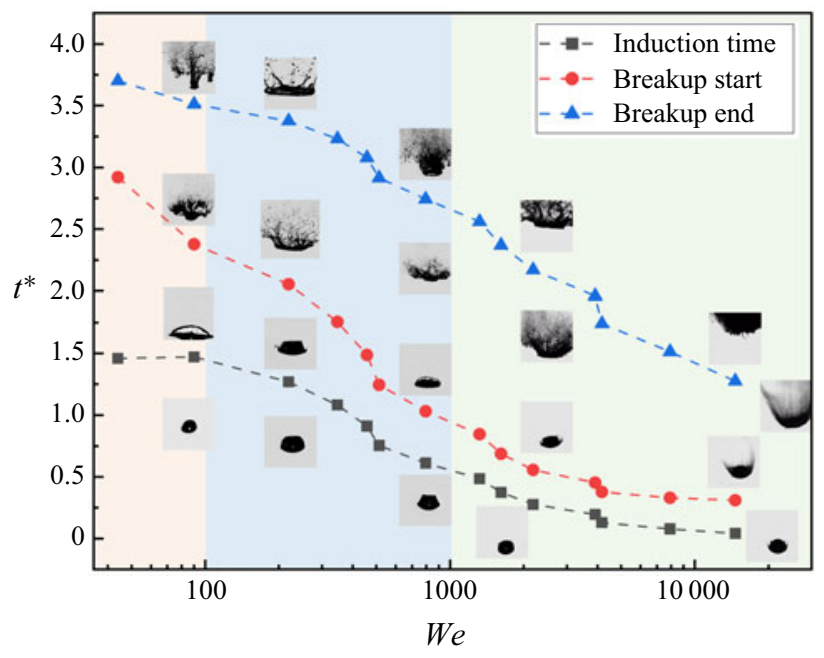

Figure 20. Regime map for shock-droplet interaction. Orange region corresponds to RTP (We $<100$ ), green region corresponds to SIE $(W e>1000)$ and blue region corresponds to transition regime $(100<W e<1000)$. Snapshot images corresponds to breakup stage at particular time instant and Weber number.

The wavelength of formed instability determines the number of bags formed during the droplet breakup process. The mode of droplet breakup is classified by normalising the initial droplet diameter with RT wavelength $\left(N=D_{i} / \lambda_{R T}\right)$ (Zhao et al. 2010). In the present work, $N \sim 2-3$ for all considered cases (see supplementary table 1) which undergoes breakup through RTI. Therefore, only the multibag mode of droplet breakup is obtained (see figure 18) (Zhao et al. 2010; Jain et al. 2015). Several bags are formed during the multibag mode of droplet breakup on the flattened droplet between the central undeformed and peripheral rims. The formed bag grows in size along the streamwise direction, reducing the sheet thickness, making it vulnerable to rupture into secondary daughter droplets. This is then followed by the rupture of the peripheral rim, which disintegrates into larger droplet sizes compared with those formed by bag breakup. The central region of the deformed droplet takes the form of a stamen-shaped ligament, which is attached to the formed bag. After bag rupture, the stamen ligament either contracts back to form a single droplet (at low Weber numbers) or further undergoes atomisation in a manner similar to the primary droplet, depending on the external aerodynamic forces. The usage of a wide range of Weber numbers (We 30-15000) in the present study essentially resulted in the observation of both RTP and SIE modes of droplet breakup and identifying the possible transition mechanism between two modes. Subsequently, the present experimental study illustrates the aspect ratio variation for different Weber numbers and droplet sizes of $2.5 \mathrm{~mm}$ and $0.5 \mathrm{~mm}$ (see figure 19). The results are shown up to the beginning of droplets stripping for the SIE breakup mode and the beginning of bag formation during the RTP mode. As can be seen from the figure, the extent of deformation for smaller droplet size (RTP breakup mode) is greater than the bigger droplet (SIE breakup). This data could be useful during the validation of future numerical models.

\section{Conclusions}

The multiscale nature of shock-droplet interaction dynamics was studied through inline shadowgraphy/schlieren imaging and top view shadowgraphy imaging. A pulsed laser 
with nanosecond-order pulse width was used, which effectively freezes the high-speed phenomena occurring during shock-droplet interaction. The experiments were carried out for a shock Mach number range of 1.1-1.8, droplet sizes of $\sim 0.5,2.5,2.9 \mathrm{~mm}$, resulting in a wide range of Weber numbers ( 30-15 000) and Reynolds numbers ( 3000-110 000). The usage of high-quality and precise experimental data in this work makes the present results an ideal benchmark for future numerical studies. The complete regime map of the droplet morphology at different time instants and Weber numbers is shown in figure 20.

The interaction dynamics was bifurcated into two stages: initial wave dynamics (stage I) and droplet breakup dynamics (stage II). Stage I includes the formation of different wave structures involving reflected waves, transmitted waves and refracted waves. The evolution dynamics of each wave structure was explained along with the local changes imposed by them on the droplet surface. The formation of high-pressure regions near the front stagnation point due to flow stagnation and at the rear stagnation point due to Mach wave focusing and recirculating jet flow results in the deformation of the droplet into a cupcake shape, which marks the end of the stage I interaction. Stage I was observed to be self-similar for all experimental cases considered in this work, irrespective of shock Mach number.

Stage II of the interaction dynamics corresponds to the droplet breakup. Two modes of droplet breakup, i.e. SIE breakup (based on KHI) and RTP breakup (based on RTI), were observed. The SIE mode is triggered by the formation of $\mathrm{KH}$ waves on the windward surface of the droplet. The formed waves grow in size as they translate after formation and merge at the equator, forming a thin sheet that undergoes rupture and results in daughter droplets/mist generation. The mechanism of daughter droplet formation based on the relative dominance of droplet deformation and $\mathrm{KH}$ wave formation in liquid transport was used to explain the monotonic increase in droplet size with time. The same argument was also used for explaining the decrease in the daughter droplet sizes with an increase in Weber number. The top view of interaction dynamics shows the periodic distribution of formed liquid ligament during the initial breakup stage, which was explained using the transverse modulation of RT waves on the surface of $\mathrm{KH}$ waves. The comparison of theoretical prediction of observed $\mathrm{KH}$ waves and transverse modulated waves shows a qualitatively good agreement. However, quantitatively, the predicted results match only in orders of magnitude, and possible means for this discrepancy were also discussed. The droplet breakup through the SIE mechanism was found to be recurrent. The formed sheet near the droplet equator undergoes holes nucleation at several locations, and the growth of formed holes leads to the rupture of the sheet into daughter droplet/mist in later instances. This cycle of sheet formation and rupture into daughter droplets continues until complete disintegration of the droplet or if the external aerodynamic forces cannot assist until further breakup.

The RTP-based droplet breakup is characterised by the complete deformation of the primary droplet into a flattened disc. The windward surface of the formed disc experiences an acceleration of low density induced airflow, making it susceptible to RT-based instability waves. A comparison of the theoretical model based on RTI predicts a multibag type of droplet breakup for all considered RTP cases, which matches well with the experiments. The result shows a KH wave growth rate dominance over RT waves, indicating $\mathrm{KH}$ waves as a primary mechanism for droplet breakup. The RT waves can only form when $\mathrm{KH}$ wave formation is unfavourable. Therefore, a criterion for breakup mode transition from KHI to RTP was obtained based on the ratio of droplet diameter to the wavelength of the KH wave. It was shown that KH-based SIE exist only if $D_{i} \gg \lambda_{K H}$ and transition to RTP occurs when $D_{i} \sim \lambda_{K H}$. The experimental results clearly reveal the 


\section{S. Sharma, A.P. Singh, S.S. Rao, A. Kumar and S. Basu}

plausible variation in surface flow features. Characterisation of a wide range of Weber numbers unearths the essential criteria for convenient atomisation of the liquid droplets.

Supplementary material and movies. Supplementary material and movies are available at https://doi.org/ 10.1017/jfm.2021.860.

Acknowledgements. The authors gratefully acknowledge Professor C. Tropea (TU Darmstadt, Germany), Professor S. Chakraborty (IIT KGP, India), Professor I. Roisman (TU Darmstadt, Germany), SMS group (Germany), Tata steel (India) and IGSTC PPAM group for their insights and discussions during the development of the present work.

Funding. The support of research by IGSTC (Indo-German Science and Technology Center) through project no. SP/IGSTC-18-0003 is thankfully acknowledged.

Declaration of interest. The authors report no conflict of interest.

Author ORCIDs.

(1) Shubham Sharma https://orcid.org/0000-0002-8704-887X;

Awanish Pratap Singh https://orcid.org/0000-0003-1374-8176;

S. Srinivas Rao https://orcid.org/0000-0003-1817-5207;

Aloke Kumar https://orcid.org/0000-0002-7797-8336;

Saptarshi Basu https://orcid.org/0000-0002-9652-9966.

\section{REFERENCES}

Anderson, J.D. 2003 Modern Compressible Flow: With Historical Perspective, 7th edn. McGraw-Hill.

BAYVEL, L.P. 1993 Liquid Atomization, vol. 1040. CRC.

BEN-Dor, G. 2007 Shock Wave Reflection Phenomena. Springer.

Beresh, S., et al. 2015 Pulse-burst PIV in a high-speed wind tunnel. Meas. Sci. Technol. 26 (9), 095305.

BiAsioRi-Poulanges, L. \& El-RABII, H. 2019 High-magnification shadowgraphy for the study of drop breakup in a high-speed gas flow. Opt. Lett. 44 (23), 5884-5887.

CAO, X.-K., Sun, Z.-G., LI, W.-F., LiU, H.-F. \& YU, Z.-H. 2007 A new breakup regime of liquid drops identified in a continuous and uniform air jet flow. Phys. Fluids 19 (5), 057103.

Chang, C.-H., Deng, X. \& Theofanous, T.G. 2013 Direct numerical simulation of interfacial instabilities: a consistent, conservative, all-speed, sharp-interface method. J. Comput. Phys. 242, 946-990.

CHEN, H. 2008 Two-dimensional simulation of stripping breakup of a water droplet. AIAA J. 46 (5), $1135-1143$.

Chou, W.-H., Hsiang, L.-P. \& Faeth, G.M. 1997 Temporal properties of drop breakup in the shear breakup regime. Intl J. Multiphase Flow 23 (4), 651-669.

Das, P. \& UdAYKumar, H.S. 2020 A sharp-interface method for the simulation of shock-induced vaporization of droplets. J. Comput. Phys. 405, 109005.

Dorschner, B., Biasiori-Poulanges, L., Schmidmayer, K., El-Rabit, H. \& Colonius, T. 2020 On the formation and recurrent shedding of ligaments in droplet aerobreakup. J. Fluid Mech. 904, A20.

Drazin, P.G. \& ReID, W.H. 2004 Hydrodynamic Stability, 2nd edn. Cambridge University Press.

Eggers, J. \& Villermaux, E. 2008 Physics of liquid jets. Rep. Prog. Phys. 71 (3), 036601.

Fedotov-Gefen, A., Efimov, S., Gilburd, L., Gleizer, S., Bazalitsky, G., Gurovich, V.T. \& KRASIK, Y.E. 2010 Extreme water state produced by underwater wire-array electrical explosion. Appl. Phys. Lett. 96 (22), 221502.

Guan, B., Liu, Y., Wen, C.-Y. \& Shen, H. 2018 Numerical study on liquid droplet internal flow under shock impact. AIAA J. 56 (9), 3382-3387.

Guildenbecher, D.R., LóPez-Rivera, C. \& Sojka, P.E. 2009 Secondary atomization. Exp. Fluids 46, $371-402$.

HAN, J. \& TRYgGvason, G. 2001 Secondary breakup of axisymmetric liquid drops. II. Impulsive acceleration. Phys. Fluids 13 (6), 1554-1565.

Havermann, M., Haertig, J., Rey, C. \& George, A. 2008 PIV measurements in shock tunnels and shock tubes. Top. Appl. Phys. 112, 429-443.

HENDERSON, L.F. 1989 On the refraction of shock waves. J. Fluid Mech. 198, 365-386.

HINZE, J.O. 1955 Fundamentals of the hydrodynamic mechanism of splitting in dispersion processes. AIChE J. 1 (3), 289-295. 


\section{Shock induced aerobreakup of a droplet}

HSIANG, L.-P. \& FAETH, G.M. 1992 Near-limit drop deformation and secondary breakup. Intl J. Multiphase Flow 18 (5), 635-652.

IGRA, D. \& TAKAYAMA, K. 2003 Experimental investigation of two cylindrical water columns subjected to planar shock wave loading. Trans. ASME J. Fluids Engng 125, 325-331.

JACKIW, I.M. \& ASHGRIZ, N. 2021 On aerodynamic droplet breakup. J. Fluid Mech. 913, A33.

Jain, M., Prakash, R.S., Tomar, G. \& Ravikrishna, R.V. 2015 Secondary breakup of a drop at moderate Weber numbers. Proc. R. Soc. A: Math. Phys. Engng Sci. 471 (2177), 20140930.

Jalaal, M. \& Mehravaran, K. 2014 Transient growth of droplet instabilities in a stream. Phys. Fluids 26 (1), 012101.

JARRAhBASHI, D. \& SiRIgnANO, W.A. 2014 Vorticity dynamics for transient high-pressure liquid injection. Phys. Fluids 26 (10), 101304.

Jarrahbashi, D., Sirignano, W.A., Popov, P.P. \& Hussain, F. 2016 Early spray development at high gas density: hole, ligament and bridge formations. J. Fluid Mech. 792, 186-231.

Joseph, D.D., Belanger, J. \& Beavers, G.S. 1999 Breakup of a liquid drop suddenly exposed to a high-speed airstream. Intl J. Multiphase Flow 25 (6-7), 1263-1303.

Keshavarz, B., Houze, E.C., Moore, J.R., Koerner, M.R. \& McKinley, G.H. 2016 Ligament mediated fragmentation of viscoelastic liquids. Phys. Rev. Lett. 117 (15), 154502.

Kim, D., Desjardins, O., Herrmann, M. \& Moin, P. 2006 Toward two-phase simulation of the primary breakup of a round liquid jet by a coaxial flow of gas. In Center for Turbulence Research, Annual Research Briefs, pp. 185-195. Stanford University.

KRZECZKOWSKI, S.A. 1980 Measurement of liquid droplet disintegration mechanisms. Intl J. Multiphase Flow 6 (3), 227-239.

Lefebvre, A.H. \& MCDonell, V.G. 2017 Atomization and Sprays. CRC.

LiANG, Y., JiAnG, Y., WEN, C.-Y. \& LiU, Y. 2020 Interaction of a planar shock wave and a water droplet embedded with a vapour cavity. J. Fluid Mech. 885, R6.

LiEPMANN, D. \& GHARIB, M. 1992 The role of streamwise vorticity in the near-field entrainment of round jets. J. Fluid Mech. 245, 643-668.

LiU, N., WANG, Z., SUN, M., WANG, H. \& WANG, B. 2018 Numerical simulation of liquid droplet breakup in supersonic flows. Acta Astron. 145, 116-130.

Liverts, M., Ram, O., SAdot, O., Apazidis, N. \& Ben-Dor, G. 2015 Mitigation of exploding-wire-generated blast-waves by aqueous foam. Phys. Fluids 27 (7), 076103.

Marmottant, P. \& Villermaux, E. 2004 On spray formation. J. Fluid Mech. 498, 73-111.

Mehta, Y., JAckson, T.L., Zhang, J. \& Balachandar, S. 2016 Numerical investigation of shock interaction with one-dimensional transverse array of particles in air. J. Appl. Phys. 119 (10), 104901.

Mehta, Y., Neal, C., Salari, K., Jackson, T.L., Balachandar, S. \& Thakur, S. 2018 Propagation of a strong shock over a random bed of spherical particles. J. Fluid Mech. 839, 157-197.

Meng, J.C. \& Colonius, T. 2015 Numerical simulations of the early stages of high-speed droplet breakup. Shock Waves 25 (4), 399-414.

Nicholls, J.A. \& RANGER, A.A. 1969 Aerodynamic shattering of liquid drops. AIAA J. 7 (2), $285-290$.

PILCH, M. \& ERDMAN, C.A. 1987 Use of breakup time data and velocity history data to predict the maximum size of stable fragments for acceleration-induced breakup of a liquid drop. Intl J. Multiphase Flow 13 (6), 741-757.

Poplavski, S.V., Minakov, A.V., Shebeleva, A.A. \& Boyko, V.M. 2020 On the interaction of water droplet with a shock wave: experiment and numerical simulation. Intl J. Multiphase Flow 127, 103273.

RAYleigh, LoRd 1879 On the stability, or instability, of certain fluid motions. Proc. Lond. Math. Soc. s1-11 (1), 57-72.

Sembian, S., Liverts, M., Tillmark, N. \& Apazidis, N. 2016 Plane shock wave interaction with a cylindrical water column. Phys. Fluids 28 (5), 056102.

Sharma, S., Singh, A.P. \& BASU, S. 2021 On the dynamics of vortex-droplet co-axial interaction: insights into droplet and vortex dynamics. J. Fluid Mech. 918, 1-36.

Shinjo, J. \& UMEMURA, A. 2011 Surface instability and primary atomization characteristics of straight liquid jet sprays. Intl J. Multiphase Flow 37 (10), 1294-1304.

SRIDHARAN, P., JACKSON, T.L., ZhANG, J. \& BALACHANDAR, S. 2015 Shock interaction with one-dimensional array of particles in air. J. Appl. Phys. 117 (7), 075902.

Sridharan, P., Jackson, T.L., Zhang, J., Balachandar, S. \& Thakur, S. 2016 Shock interaction with deformable particles using a constrained interface reinitialization scheme. J. Appl. Phys. 119 (6), 064904.

Sun, M., Saito, T., TAKayama, K. \& TAnno, H. 2005 Unsteady drag on a sphere by shock wave loading. Shock Waves 14 (1), 3-9. 


\section{S. Sharma, A.P. Singh, S.S. Rao, A. Kumar and S. Basu}

TAnno, H., Iтон, K., SAito, T., AвE, A. \& TAкAyama, K. 2003 Interaction of a shock with a sphere suspended in a vertical shock tube. Shock Waves 13, 191-200.

Theofanous, T.G. 2011 Aerobreakup of newtonian and viscoelastic liquids. Annu. Rev. Fluid Mech. 43 (1), 661-690.

Theofanous, T.G. \& Li, G.J. 2008 On the physics of aerobreakup. Phys. Fluids 20 (5), 052103.

Theofanous, T.G., Mitkin, V.V., Ng, C.L., Chang, C-H., Deng, X. \& Sushchikh, S. 2012 The physics of aerobreakup. II. Viscous liquids. Phys. Fluids 24 (2), 022104.

Villermaux, E. 1998 Mixing and spray formation in coaxial jets. J. Propul. Power 14 (5), 807-817.

Wang, Z., Hopfes, T., Giglmaier, M. \& AdAmS, N.A. 2020 Effect of mach number on droplet aerobreakup in shear stripping regime. Exp. Fluids 61, 193.

YANG, H. \& PENG, J. 2019 Numerical study of the shear-thinning effect on the interaction between a normal shock wave and a cylindrical liquid column. Phys. Fluids 31 (4), 043101.

Zandian, A., Sirignano, W.A. \& Hussain, F. 2017 Planar liquid jet: early deformation and atomization cascades. Phys. Fluids 29 (6), 062109.

Zandian, A., Sirignano, W.A. \& Hussain, F. 2019a Length-scale cascade and spread rate of atomizing planar liquid jets. Intl J. Multiphase Flow 113, 117-141.

ZANDiAn, A., SiRignANo, W.A. \& Hussain, F. $2019 b$ Vorticity dynamics in a spatially developing liquid jet inside a co-flowing gas. J. Fluid Mech. 877, 429-470.

Zhао, H., LiU, H.-F., Li, W.-F. \& XU, J.-L. 2010 Morphological classification of low viscosity drop bag breakup in a continuous air jet stream. Phys. Fluids 22 (11), 114103.

ZhaO, H., LIU, H.-F., XU, J.-L., LI, W.-F. \& CHENG, W. 2012 Breakup and atomization of a round coal water slurry jet by an annular air jet. Chem. Engng Sci. 78, 63-74.

ZhaO, H., LiU, H.-F., XU, J.-L., Li, W.-F. \& Lin, K.-F. 2013 Temporal properties of secondary drop breakup in the bag-stamen breakup regime. Phys. Fluids 25 (5), 054102.

Zhaо, H., Wu, Z.-W., LI, W.-F., XU, J.-L. \& LiU, H.-F. 2018 Transition weber number between surfactant-laden drop bag breakup and shear breakup of secondary atomization. Fuel 221, 138-143. 